\title{
Analog Image Modeling for 3D Single Image Super Resolution and Pansharpening
}

\author{
Richard Lartey ${ }^{1}$, Weihong Guo ${ }^{1 *}$, Xiaoxiang Zhu ${ }^{2}$ and Claas Grohnfeldt ${ }^{3}$ \\ ${ }^{1}$ Department of Mathematics, Applied Mathematics and Statistics, Case Western Reserve University, Cleveland, $\mathrm{OH}$, \\ United States, ${ }^{2}$ German Aerospace Center (DLR), Wessling, Germany, ${ }^{3}$ Department of Aerospace and Geodesy, Technical \\ University of Munich, Munich, Germany
}

OPEN ACCESS

Edited by:

Ke Shi,

Old Dominion University, United States

Reviewed by:

Jian Lu,

Shenzhen University, China

Xin Guo,

Hong Kong Polytechnic University,

Hong Kong

*Correspondence:

Weihong Guo

wxg49@case.edu

Specialty section:

This article was submitted to Mathematics of Computation and

Data Science,

a section of the journal

Frontiers in Applied Mathematics and

Statistics

Received: 01 February 2020

Accepted: 11 May 2020

Published: 12 June 2020

Citation:

Lartey R, Guo W, Zhu X and Grohnfeldt C (2020) Analog Image

Modeling for 3D Single Image Super Resolution and Pansharpening.

Front. Appl. Math. Stat. 6:22 doi: 10.3389/fams.2020.00022
Image super-resolution is an image reconstruction technique which attempts to reconstruct a high resolution image from one or more under-sampled low-resolution images of the same scene. High resolution images aid in analysis and inference in a multitude of digital imaging applications. However, due to limited accessibility to high-resolution imaging systems, a need arises for alternative measures to obtain the desired results. We propose a three-dimensional single image model to improve image resolution by estimating the analog image intensity function. In recent literature, it has been shown that image patches can be represented by a linear combination of appropriately chosen basis functions. We assume that the underlying analog image consists of smooth and edge components that can be approximated using a reproducible kernel Hilbert space function and the Heaviside function, respectively. We also extend the proposed method to pansharpening, a technology to fuse a high resolution panchromatic image with a low resolution multi-spectral image for a high resolution multi-spectral image. Various numerical results of the proposed formulation indicate competitive performance when compared to some state-of-the-art algorithms.

Keywords: super-resolution, reproducible kernel Hilbert space (RKHS), heaviside, sparse representation, multispectral imaging

\section{INTRODUCTION}

During image acquisition, we often loose resolution due to the limited density of the imaging sensors and the blurring of the acquisition lens. Image super resolution, a post process to increase image resolution, is an efficient way to achieve a resolution beyond what the hardware offers. A detailed overview of this area is outlined in Farsiu et al. [1], Yang et al. [2], and Park et al. [3]. Current techniques of solving this ill-posed problem are often insufficient for many real world applications $[4,5]$. We propose an analog modeling based three-dimensional (3D) color image super resolution and pansharpening method.

In recent literature, image super-resolution (SR) methods typically fall into three categories: interpolation based methods, learning based methods, and constrained reconstruction methods. Interpolation based methods $[6,7]$ such as nearest-neighbor, bilinear and bicubic interpolation use analytical priors to recover a continuous-time signal from the discrete pixels. They work very well for smooth regions and are simple to compute. In dictionary learning based models, the missing high frequency detail is recovered from a sparse linear combination of dictionary atoms trained from patches of the given low-resolution (LR) image [8,9] or from an external database of patches [10-12]. Dictionary learning based methods explore the self-similarities of the image and 
are robust, albeit rather slow training time of the dictionary atoms. Models based on constrained reconstruction use the underlying nature of the LR image to define prior constraints for the target HR image. Sharper structures can be recovered using these models. Priors common to these techniques include total variation priors [13-15], gradient priors [16, 17] and priors based on edge statistics [18]. The proposed method falls in this category. Another category is deep neural network based approach that uses a large amount of training data to obtain super results [19-22].

Increased accessibility to satellite data has led to a wide range of applications in multispectral (MS) SR. These applications include target detection, scene classification, and change detection. A multitude of algorithms have been presented in recent literature to address this task. However, the performance of many of these algorithms are data dependent with respect to existing quantitative assessment measures. With the increasing advancements in technology, there have been varying modes of image acquisition systems. In remote sensing, satellites carry sensors that acquire images of a scene in multiple channels across the electromagnetic spectrum. This gives rise to what is referred to as multispectral imaging. Due to high cost overheads in developing sensors that can faithfully acquire scene data with high spectral and spatial resolution, the spatial resolution in the data acquisition process is often compromised. The lowspatial resolution MS images usually consist of images across 4-8 spectral bands: red, green, blue, as well as near infrared bands (provide Landsat, IKONOS, etc. specifications).

Most satellites also acquire a high-spatial resolution image known as a panchromatic image. The technique of using panchromatic (PAN) to compensate for the reduction in spatialresolution of the MS images is referred to as pansharpening. The success of pansharpening relies on the extent one can explore the structural similarity between the PAN and the high-resolution (HR) MS image [23]. In recent years, pansharpening methods fall under three main categories: component substitituion (CS), multiresolution analysis (MRA), and variational methods. Detailed surveys can be found in Loncan et al. [24] and Vivone et al. [25].

The main assumption of CS methods is that the spatial and spectral information of the high spectral resolution image can be separated in some space [24]. Examples of CS methods include principal component analysis (PCA) [26, 27], intensityhue-saturation (IHS) methods [28-30], Brovey method [31], and Gram-Schmidt (GS) based methods [32]. These methods enhance the spatial resolution of the LR MS image at the expense of some loss in the spectral detail. Compared to the CS methods, MRA methods have better temporal coherence between the PAN and the enhanced MS image. MRA methods yield results with better spectral consistency at the expense of designing complex filters to mimic the reduction in the resolution by the sensors. Spatial details are injected into the MS data after a multi-scale decomposition of the PAN [24]. Examples of MRA methods include high-pass filtering (HPF) [30], smooth filterbased intensity modulation (SFIM) [33], generalized Laplacian pyramid (GLP) methods [34, 35], additive "á trous" wavelet transform (ATWT) methods [23, 36] and additive wavelet luminance proportional (AWLP) [37]. Variational methods are more robust compared to the CS and MRA techniques, albeit rather slow in comparison [24]. A common tool used by these approaches is regularization. This is used to promote some structural similarity between the LR MS, HR MS, and PAN images $[24,38,39]$. Among these methods include models based on the Bayesian framework and matrix factorization methods [24, 38, 39].

In this paper, we extend the key ideas in the SR model in Deng et al. [40] to 3D framework. We then test the performance of the model with single image super-resolution and pansharpening.

\subsection{Related Work}

In Deng et al. [40], a 2D image defined on a continuous domain $[0,1] \times[0,1]$ is assumed to have a density function that is the sum of two parts, reproducing kernel Hilbert space (RKHS) and Heaviside functions to study single image super-resolution (SISR) whereby the problem is cast as an image intensity function estimation problem. They assume that the smooth components of the image belong to a special Hilbert space called RKHS, and can be spanned by a basis based on an approximation by spline interpolation [41]. The Heaviside function is used to model the edges and discontinuous information in the input image. Using a patch-based approach, the intensity information of the LR input image patches are defined on a coarser grid to estimate the coefficients of the basis functions. To obtain the enhanced resolution result, they utilize the obtained coefficients and an approximation of the basis functions on a finer grid. Furthermore, to recover more high frequency information the authors employ an iterative back projection method [42] after the HR image has been obtained. Color images were tested in Deng et al. [40], however the analog modeling was done the luminance channel only after transforming the color space. This RKHS+Heaviside method is extended to pansharpening in Deng et al. [43]. In the pansharpening framework the different bands share the same basis but with different coefficients patch by patch, and channel by channel. A panchromatic image is used to guide the model and it is assumed to be a linear combination of the multiple bands.

\subsection{Contribution}

Using the same basis as in Deng et al. [40], we present a three-dimensional SR algorithm for true color images based on continuous/analog modeling. We also extend it to pansharpening. Instead of estimating the basis coefficients patch by patch and band by band, we cluster patches and conduct computation cluster by cluster. Similarity of patches within one cluster leads to some natural regularity. We jointly optimize all the coefficients for all the bands of the model rather than optimizing for each independent band. Furthermore, we use clustering techniques to improve the structural coherence of the desired results in the optimization model. Spatially, we divide the images into small overlapping patches and cluster these patches classes using k-means clustering. To improve the spectral coherence of the results, we cluster the image bands into groups based on correlation statistics and the perform the optimization on each of the groups obtained. 
In this paper, we use the RKHS approximations to model the smooth components of the image whiles preserving the nonsmooth components by the approximated Heaviside function. For the pansharpening experiments, We also preprocess the data using fast state-of-the-art MRA techniques [37] to incorporate some similarity in the spectral channels of the fused image. Thus we develop a continuous 3D modeling framework for multispectral pansharpening.

The experiments that follow show that these contributions yield better enhanced resolution results with faster convergence speeds at all scales.

The paper is organized as follows. In section 2, we review the regular functions used in decomposing the image. The proposed model is described in section 3 with numerical results outlined in section 4 .

\section{BACKGROUND REVIEW}

In this section, we review the mathematical functions used in the formulation of our model. Similar as in Deng et al. [40], we model image intensity function defined on a small image patch as a linear combination of reproducing Kernel Hilbert function (RKHS) and approximated Heaviside function (AHF) which models the smooth and edge components, respectively.

\subsection{Reproducible Kernel Hilbert Spaces}

RKHS can be used to define feature spaces to help compare objects that have complex structures and are hard to distinguish in the original feature space. Wahba [41] proposed polynomial spline models for smoothing problems. Our proposed model is based on this approach using the Taylor series expansion. We review these methods in the following subsections.

\subsubsection{Signal Smoothing Using Splines}

Let $\mathcal{G}$ represent a family of functions on $[0,1]$ with continuous derivatives up to order $(m-1)$,

$$
\mathcal{G}=\left\{f: f \in \mathcal{C}^{m-1}[0,1], f^{(m)} \in \mathcal{L}_{2}[0,1]\right\} .
$$

By Taylor's theorem with remainder for $f \in \mathcal{G}$, we may write

$$
f(t)=\underbrace{\sum_{\nu=0}^{m-1} \frac{t^{v}}{v !} f^{(v)}(0)}_{f_{0}(t)}+\underbrace{\int_{0}^{1} \frac{(t-u)_{+}^{m-1}}{(m-1) !} f^{(m)}(u) d u}_{f_{1}(t)},
$$

for some $u$, where $(x)_{+}=x$ for $x \geq 0$ and $(x)_{+}=0$ otherwise. Let

$$
\phi_{v}(t)=\frac{t^{v-1}}{(v-1) !}, \quad 1 \leq v \leq m
$$

and $\mathcal{H}_{0}=\operatorname{span}\left\{\phi_{i}\right\}_{1 \leq i \leq m}$ endowed with norm $\|\phi\|^{2}=$ $\sum_{\nu=0}^{m-1}\left[\left(D^{v} \phi\right)(0)\right]^{2}$, then $D^{(m)}\left(\mathcal{H}_{0}\right)=0$, where $D^{m}$ denotes the $m$ th derivative. It can be shown that a reproducing kernel exists for $\mathcal{H}_{0}$ and is defined as

$$
R^{0}(s, t)=\sum_{\nu=1}^{m} \phi_{v}(s) \phi_{v}(t)
$$

therefore for any $f_{0} \in \mathcal{H}$, we have $f_{0}(t)=\sum_{\nu=1}^{m} d_{\nu} \phi_{\nu}(t)$.

Let $\mathcal{B}_{m}=\left\{f: f \in \mathcal{C}^{m-1}[0,1], f^{\nu}(0)=0, v=0,1, \ldots, m-1\right\}$ and define

$$
G_{m}(t, u)=\frac{(t-u)_{+}^{m-1}}{(m-1) !}
$$

The space

$$
\begin{aligned}
\mathcal{H}_{1}=\left\{f: f \in \mathcal{B}_{m}, \text { with } f, f^{\prime}, \ldots, f^{(m-1)}\right. \\
\\
\left.\quad \text { absolutely continuous, } f^{(m)} \in \mathcal{L}_{2}\right\}
\end{aligned}
$$

with norm $\|f\|^{2}=\int_{0}^{1}\left(f^{(m)}(t)\right)^{2} d t$ can be shown to be an RKHS with reproducing kernel

$$
R^{1}(x, t)=\int_{0}^{1} G_{m}(t, u) G_{m}(x, u) d u,
$$

so that for any $f_{1} \in \mathcal{H}_{1}$, we have $f_{1}(t)=\sum_{i=1}^{n} c_{i} \xi_{i}$, where $\xi_{i}(\cdot)=R^{1}\left(s_{i}, \cdot\right)$. It follows from the properties of the RKHS, we can construct a direct sum space $\mathcal{G}_{m}=\mathcal{H}_{0}+\mathcal{H}_{1}$ since

$$
\int_{0}^{1}\left(\left(D^{m} f_{0}\right)(u)\right)^{2} d u=0, \quad \sum_{\nu=0}^{m-1}\left(D^{(v)} f_{1}(0)\right)^{2}=0 .
$$

The reproducing kernel for $\mathcal{G}_{m}$ can be shown [41] to be

$$
R(s, t)=R^{0}(s, t)+R^{1}(s, t)
$$

with norm

$$
\|f\|^{2}=\sum_{\nu=0}^{m-1}\left[\left(D^{\nu} f\right)(0)\right]^{2}+\int_{0}^{1}\left(f^{(m)}(t)\right)^{2} d t, \quad f \in \mathcal{G}_{m},
$$

therefore for any $f \in \mathcal{G}_{m}$, we have $f=f_{0}+f_{1}$ with $f_{0} \in \mathcal{H}_{0}, f_{1} \in$ $\mathcal{H}_{1}$ and

$$
f(t)=\sum_{\nu=1}^{m} d_{\nu} \phi_{\nu}(t)+\sum_{i=1}^{n} c_{i} \xi_{i}, \quad t \in[0,1]
$$

Let $\boldsymbol{d}=\left(d_{1}, \ldots d_{m}\right)^{T}$ and $\boldsymbol{c}=\left(c_{1}, \ldots, c_{n}\right)^{T}$ be coefficient vectors, $\boldsymbol{f}=\left(f\left(t_{1}\right), \ldots, f\left(t_{p}\right)\right)^{T}$ represent the discretization of $f$ on discrete grids $t_{j}, j=1,2, \cdots n_{t}, \boldsymbol{T} \in \mathbb{R}^{n \times m}, \boldsymbol{\Sigma} \in \mathbb{R}^{n \times n}$ with $\boldsymbol{T}_{j v}=\phi_{v}\left(t_{j}\right), \boldsymbol{\Sigma}_{j i}=\xi_{i}\left(t_{j}\right)$, then $\boldsymbol{f}=\boldsymbol{T} \boldsymbol{d}+\boldsymbol{\Sigma} \boldsymbol{c}$. Given the observation data

$$
g=f+\eta
$$

where $\boldsymbol{\eta}$ denotes additive Gaussian noise. To find $\boldsymbol{f}$ from $\boldsymbol{g}$, we need to estimate the coefficients $\boldsymbol{d}$ and $\boldsymbol{c}$. Following Wahba [41], the coefficients $d, c$ are estimated from the discrete measurements $g$ by

$$
(\boldsymbol{c}, \boldsymbol{d})=\arg \min \left\{\frac{1}{n}\|\boldsymbol{g}-\boldsymbol{T} \boldsymbol{d}-\boldsymbol{\Sigma} \boldsymbol{c}\|^{2}+\mu \boldsymbol{c}^{T} \boldsymbol{\Sigma} \boldsymbol{c}\right\},
$$

where the term $\mu \boldsymbol{c}^{T} \boldsymbol{\Sigma} \boldsymbol{c}$ is a non-smoothness penalty. Closed form solutions for $\boldsymbol{c}$ and $\boldsymbol{d}$ can be obtained. The parameter $m$ controls the total degree of the polynomials used to define $\boldsymbol{T}$. For example, when $m=3$ the basis functions are given as $\phi_{1}(x)=1, \phi_{2}(x)=x$ and $\phi_{3}(x)=x^{2} / 2$. 


\subsubsection{Image Smoothing Using Splines}

Extending the one-dimensional spline model to two-dimensional data, we consider a similar discretization and set up a model similar to that defined in (11). Let $\boldsymbol{f}=\left(f\left(t_{1}\right), \ldots, f\left(t_{n_{t}}\right)\right)^{T}$, $t_{i} \in[0,1] \times[0,1]$ be a discretization for an observed noisy image. From Wahba [41], an estimate of $\boldsymbol{f}$ can be obtained from the additive noise data model in (10) for images by minimizing the following

$$
\min \left\{\frac{1}{n}\|\boldsymbol{g}-\boldsymbol{f}\|_{2}^{2}+\mu J_{m}(\boldsymbol{f})\right\}
$$

where $m$ defines the order of the partial derivatives in $\mathcal{L}_{2}([0,1] \times$ $[0,1])$, and with the penalty function defined by

$$
J_{m}(\boldsymbol{f})=\sum_{\nu=0}^{m} \int_{0}^{1} \int_{0}^{1}\left(\begin{array}{l}
m \\
v
\end{array}\right)\left(\frac{\partial^{m} f}{\partial x^{v} \partial y^{m-v}}\right)^{2} d x d y
$$

The null space of $J_{m}$ is the $M=\left(\begin{array}{c}2+m-1 \\ 2\end{array}\right)$-dimensional space spanned by polynomials in two variables with total degree at most $m-1$. In this paper, and we choose $m=3$ so that $M=6$ and the null space of $J_{m}$ is spanned by monomials $\phi_{1}, \phi_{2}, \ldots, \phi_{6}$ given by

$$
\begin{array}{r}
\phi_{1}(x, y)=1, \phi_{2}(x, y)=x, \phi_{3}(x, y)=y, \\
\phi_{4}(x, y)=x y, \phi_{5}(x, y)=x^{2}, \text { and } \phi_{6}(x, y)=y^{2} .
\end{array}
$$

Duchon [44] proved that a unique minimizer $f_{\mu}$ exists for (12) with representation

$$
f_{\mu}(t)=\sum_{\nu=1}^{M} d_{\nu} \phi_{\nu}(t)+\sum_{i=1}^{n} c_{i} E_{m}\left(t, t_{i}\right), \quad t \in[0,1] \times[0,1]
$$

where $E_{m}$ is defined as

$$
E_{m}(s, t)=E_{m}(|s-t|)=\theta_{m, d}|s-t|^{2 m-d} \ln |s-t|,
$$

and $\theta_{m, d}=\frac{(-1)^{d / 2+m+1}}{2^{2 m-1} \pi^{d / 2}(m-1) !(m-d / 2) !}$.

Based on the work of Duchon [44] and Meinguet [45] we can rewrite (12) to find minimizers $c$ and $\boldsymbol{d}$ by

$$
(\boldsymbol{c}, \boldsymbol{d})=\arg \min \left\{\|\boldsymbol{g}-\boldsymbol{T} \boldsymbol{d}-\boldsymbol{K} \boldsymbol{c}\|^{2}+\mu \boldsymbol{c}^{T} \boldsymbol{K} \boldsymbol{c}\right\}
$$

where $\boldsymbol{T} \in \mathbb{R}^{n_{t} \times M}$, with $\boldsymbol{T}_{i, v}=\phi_{v}\left(t_{i}\right)$ and $\boldsymbol{K} \in \mathbb{R}^{n_{t} \times n_{t}}$ with $\boldsymbol{K}_{i, j}=E_{m}\left(t_{i}, t_{j}\right) . E_{m}(s, t)$ is the two-dimensional equivalent of $\xi_{i}(t)$ in the one-dimensional case. After obtaining the coefficients, we compute $f$ using the relationship $f=T \boldsymbol{d}+\boldsymbol{K} \boldsymbol{c}$.

\subsection{Approximated Heaviside Function}

The one-dimensional Heaviside step function is defined as

$$
\phi(x)=\left\{\begin{array}{l}
0, x<0 \\
1, x \geq 0
\end{array}\right.
$$

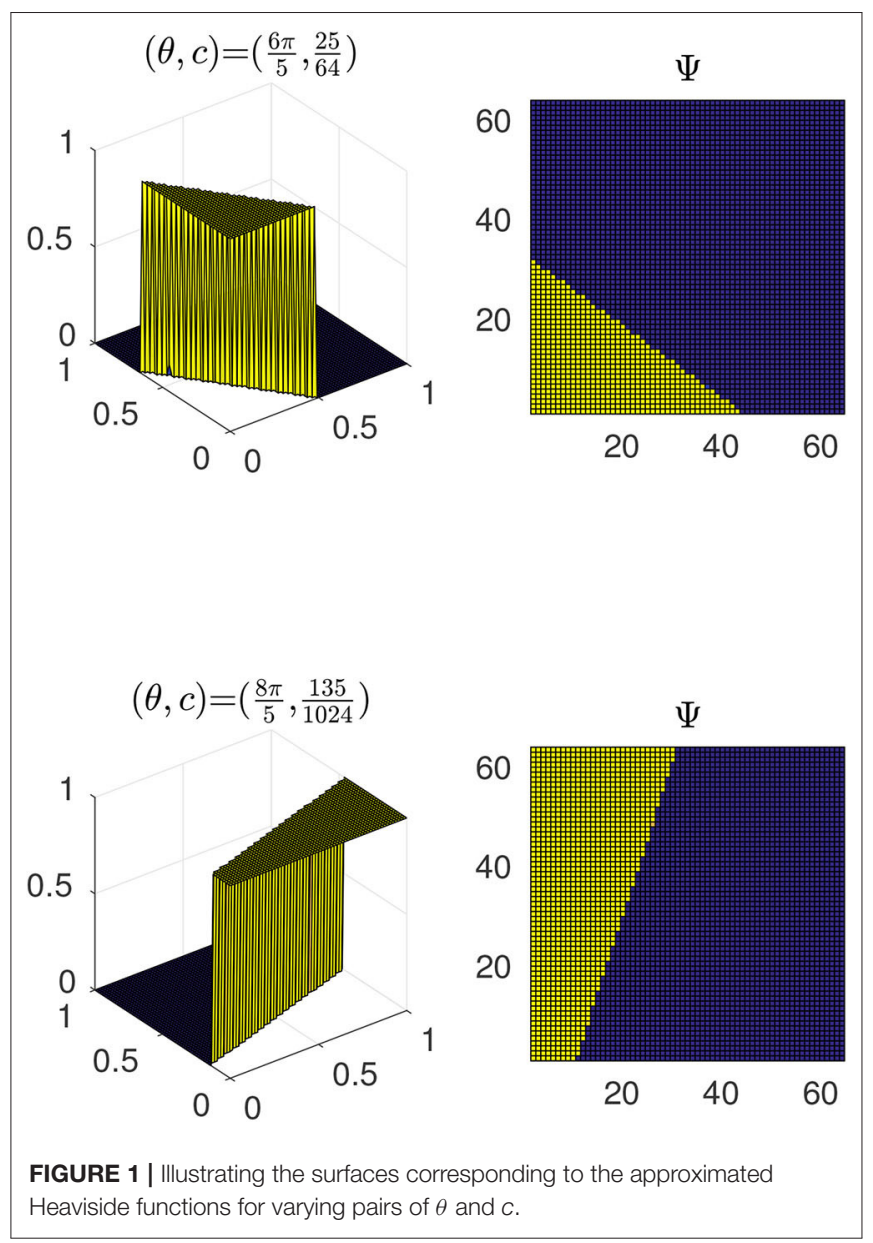

Due to the singularity at $x=0$, we approximate $\phi$ by,

$$
\psi(x)=\frac{1}{2}+\frac{1}{\pi} \arctan \left(\frac{x}{\varepsilon}\right), \quad \varepsilon \in \mathbb{R}
$$

We refer to this as the approximated Heaviside function (AHF), an approximation to $\phi(x)$ as $\varepsilon \rightarrow 0$. The variable $\varepsilon$ controls the smoothness of the approximation [40]. A two-dimensional variation of the AHF is given by

$$
\Psi(x, y)=\psi\left(\left(\begin{array}{c}
\cos \theta \\
\sin \theta
\end{array}\right) \cdot\left(\begin{array}{l}
x \\
y
\end{array}\right)+c\right)
$$

with variables $\theta$ and $c$ determining the rotations and locations of the edges as shown in Figure 1, we show the 2D and 3D surface view of the AHF at two different pairs of $\theta$ and $c$. Kainen et al. [46] proved that a function $f \in \mathcal{L}_{2}([0,1] \times[0,1])$ can be approximately represented by the weighted linear combination of approximated Heaviside functions:

$$
f(x, y)=\sum_{j=1}^{n_{\theta}} w_{j} \psi\left(\left(\begin{array}{c}
\cos \theta_{j} \\
\sin \theta_{j}
\end{array}\right) \cdot\left(\begin{array}{l}
x \\
y
\end{array}\right)+c_{j}\right) .
$$

We define $\boldsymbol{\Psi} \in \mathbb{R}^{n_{t} \times n_{\theta}}$ with $\boldsymbol{\Psi}_{i, j}=\psi\left(\left(\begin{array}{c}\cos \theta_{j} \\ \sin \theta_{j}\end{array}\right) \cdot\left(\begin{array}{c}x_{i} \\ y_{i}\end{array}\right)+c_{j}\right), t_{i}=$ $\left(x_{i}, y_{i}\right)$, and $n_{\theta}$ is the number of rotations considered. Suppose 


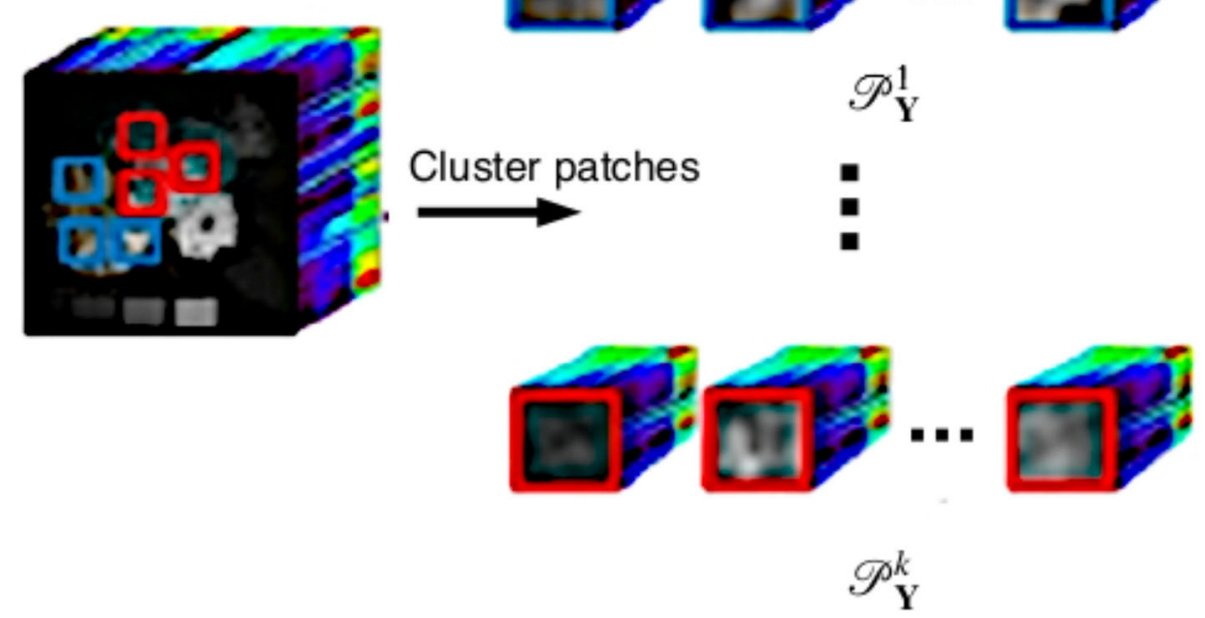

FIGURE 2 | Clustering the set of patches $\mathscr{P}_{\boldsymbol{Y}}$ into $k=2$ classes.

$f, g$ are the vectorized high and low resolution discretizations of $f$, respectively. The vectorized high resolution image $f \in$ $\mathbb{R}^{n_{1} n_{2}}$ can be obtained by $\boldsymbol{f}_{i}=f\left(t_{i}^{h}\right)$ with $t_{i}^{h}=\left(x_{i}^{h}, y_{i}^{h}\right)$, $x_{i}^{h} \in\left\{0, \frac{1}{n_{1}-1}, \frac{2}{n_{2}-1}, \ldots, 1\right\}, y_{i}^{h} \in\left\{0, \frac{1}{n_{2}-1}, \frac{2}{n_{2}-1}, \ldots, 1\right\}$ on a finer grid. Similarly, vectorized the low-resolution discretization $\boldsymbol{g} \in \mathbb{R}^{m_{1} m_{2}}$ can be obtained by $\boldsymbol{g}_{i}=f\left(t_{i}^{\ell}\right)$ with $t_{i}^{\ell}=\left(x_{i}^{\ell}, y_{i}^{\ell}\right)$, $x_{i}^{\ell} \in\left\{0, \frac{1}{m_{1}-1}, \frac{2}{m_{1}-1}, \ldots, 1\right\}$, and $y_{i}^{\ell} \in\left\{0, \frac{1}{m_{2}-1}, \frac{2}{m_{2}-1}, \ldots, 1\right\}$ on a coarse grid. Now, assuming that the underlying analog image intensity function is approximated by the sum of a RKHS function and the variations of AHF with $\boldsymbol{T}^{\ell} \in \mathbb{R}^{m_{1} m_{2} \times M}, \boldsymbol{K}^{\ell} \in$ $\mathbb{R}^{m_{1} m_{2} \times m_{1} m_{2}}$, and $\boldsymbol{\Psi}^{\ell} \in \mathbb{R}^{m_{1} m_{2} \times n_{\theta}}$, we have

$$
g=T^{\ell} d+K^{\ell} c+\Psi^{\ell} e .
$$

Thus, given the low resolution input $\boldsymbol{g}$, the coefficient vectors $\boldsymbol{c} \in \mathbb{R}^{m_{1} m_{2}}, \boldsymbol{d} \in \mathbb{R}^{M}$ and $\boldsymbol{e} \in \mathbb{R}^{n_{\theta}}$ for the residual, smooth and edge components are obtained by solving the following minimization problem:

$$
\min _{\boldsymbol{c}, \boldsymbol{d}, \boldsymbol{e}}\left\{\left\|\boldsymbol{g}-\boldsymbol{S B}\left(\boldsymbol{T}^{h} \boldsymbol{d}+\boldsymbol{K}^{h} \boldsymbol{c}+\boldsymbol{\Psi}^{h} \boldsymbol{e}\right)\right\|^{2}+\mu \boldsymbol{c}^{T} \boldsymbol{K}^{\ell} \boldsymbol{c}+\gamma\|\boldsymbol{e}\|_{1}\right\},
$$

where $\boldsymbol{B}$ is the identity matrix in the absence of blur, $S$ is the downsampling operator, and the superscripts $h$ and $\ell$ refers to a fine and course scale matrices. The smooth components of the image are modeled by the RKHS approach, while AHF caters for the edges. Since the dictionary $\boldsymbol{\Psi}$ is pretty exhaustive, i.e., accounting for multiple edge orientations, it is reasonable to use the $\|\cdot\|_{1}$ to enforces the sparsity of as all the orientations may not be present for any given image. Once the coefficients have been obtained, we have

$$
f=T^{h} d+K^{h} c+\Psi^{h} e,
$$

where $T^{h} \in \mathbb{R}^{n_{1} n_{2} \times M}, \boldsymbol{K}^{\ell} \in \mathbb{R}^{n_{1} n_{2} \times m_{1} m_{2}}$, and $\boldsymbol{\Psi}^{\ell} \in$ $\mathbb{R}^{n_{1} n_{2} \times n_{\theta}}$. Next, we discuss our proposed model and examine some numerical experiments in the chapters that follow.

\section{PROPOSED MODELS}

In this section, we present our proposed models for SISR and pansharpening based on the functions defined in section 2 . We propose a 3D patch-based approach that infers the HR patches from LR patches that has been grouped into classes based on their structural similarity. As a result, we impose similarity constraints within the classes so that the coefficients for neighboring patches in a group do not differ substantially. In addition, we use sparse constrained optimization techniques that simplify the minimization of the resulting energy functional by solving a series of subproblems, each with a closed form solution. Two algorithms will be discussed. The first is a 3D SR model for true color images and MS images with at most four bands. The second algorithm is an extension of our SISR model to pansharpening for MS images with at least four spectral bands. This is a two-step hybrid approach that incorporates CS and MRA methods into the first algorithm to obtain an enhanced resolution result.

\subsection{Single Image Super-Resolution}

Given a LR image $\boldsymbol{Y} \in \mathbb{R}^{m_{1} \times m_{2} \times \lambda}$ we estimate the target HR image $\widehat{\boldsymbol{X}} \in \mathbb{R}^{n_{1} \times n_{2} \times \lambda}$ with $n_{i}=\omega m_{i}$, where $\omega$ is a scale factor and $\lambda$ is the number of bands as follows. We begin by decomposing the LR image into a set of overlapping patches $\mathscr{P}_{Y}=\left\{\boldsymbol{Y}_{p_{i}}\right\}_{i=1}^{N_{p}}$, $\boldsymbol{Y}_{p_{i}} \in \mathbb{R}^{m_{p} \times m_{p} \times \lambda}$. The size of the square patches, and the overlap between adjacent patches depend on the dimensions of the input image. We consider the use of overlapping patches to improve local consistency of the estimated image across the regions of overlap. The estimated image $\widehat{\boldsymbol{X}}$ will be represented by the same 
number of patches, $N_{p}$. Next, for a suitably chosen $k$, we use the $k$ means algorithm with careful seeding [47] to cluster the patches into $k$ classes $\mathscr{P}_{Y}^{1}, \ldots \mathscr{P}_{Y}^{k}$, as shown in Figure 2. In this paper, the value of $k$ is determined empirically to obtain the best result. Clustering is done so that each high resolution patch generated preserves some relationships with its neighboring patches.

Following the clustering, we consider each set of patches $\mathscr{P}_{\boldsymbol{Y}}^{i}=\left\{\boldsymbol{Y}_{p_{j}}\right\}_{j \in I_{i}}$, for some index set $I_{i}$ with $1 \leq i \leq k$. Let $\boldsymbol{Y}_{\left(\lambda, I_{i}\right)} \in \mathbb{R}^{m_{p} m_{p} \times \lambda N_{I_{i}}}$ and $\widehat{\boldsymbol{X}}_{\left(\lambda, I_{i}\right)} \in \mathbb{R}^{n_{p} n_{p} \times \lambda N_{I_{i}}}$ represent the images corresponding to the set of LR and HR patches $\mathscr{P}_{Y}^{i}$ and $\mathscr{P}_{\widehat{X}}^{i}$ defined by

$$
\begin{aligned}
\boldsymbol{Y}_{\left(\lambda, I_{i}\right)} & =\left[\boldsymbol{y}_{1, p_{1}}, \ldots, \boldsymbol{y}_{\lambda, p_{1}}, \ldots, \boldsymbol{y}_{1, p_{N_{I_{i}}}}, \ldots, \boldsymbol{y}_{\lambda, p_{N_{I_{i}}}}\right] \\
\widehat{\boldsymbol{X}}_{\left(\lambda, I_{i}\right)} & =\left[\widehat{\boldsymbol{x}}_{1, p_{1}}, \ldots, \widehat{\boldsymbol{x}}_{\lambda, p_{1}}, \ldots, \widehat{\boldsymbol{x}}_{1, p_{N_{I_{i}}}}, \ldots, \widehat{\boldsymbol{x}}_{\lambda, p_{N_{I_{i}}}}\right]
\end{aligned}
$$

where $n_{p}=\omega m_{p}$ for some scale factor $\omega \in \mathbb{N}, \boldsymbol{y}_{i, p_{j}}$ and $\widehat{\boldsymbol{x}}_{i, p_{j}}$ represents the vectorization of the $i$-th band of the $j$-th patch, with $1 \leq i \leq \lambda$ and $j \in I_{i}$. Here, $N_{I_{i}}$ represents the total number of patches in the set $\mathscr{P}_{Y}^{i}$. Using the RKHS and AHF functions as in Deng et al. [40] we can find an embedding for the highdimensional data that is also structure preserving. We further assume that the HR and LR image patches have similar local geometry and are related by the equations

$$
\begin{aligned}
& \boldsymbol{Y}_{\left(\lambda, I_{i}\right)}=\boldsymbol{T}^{\ell} \boldsymbol{D}+\boldsymbol{K}^{\ell} \boldsymbol{C}+\boldsymbol{\Psi}^{\ell} \boldsymbol{E}, \\
& \widehat{\boldsymbol{X}}_{\left(\lambda, I_{i}\right)}=\boldsymbol{T}^{h} \boldsymbol{D}+\boldsymbol{K}^{h} \boldsymbol{C}+\boldsymbol{\Psi}^{h} \boldsymbol{E},
\end{aligned}
$$

where $\boldsymbol{D}=\left[\boldsymbol{d}_{1}, \ldots \boldsymbol{d}_{\lambda N_{I_{i}}}\right] \in \mathbb{R}^{M \times \lambda N_{I_{i}}}, \quad \boldsymbol{C}=\left[\boldsymbol{c}_{1}, \ldots \boldsymbol{c}_{\lambda N_{I_{i}}}\right] \in$ $\mathbb{R}^{m_{1} m_{2} \times \lambda N_{I_{i}}}$ and $\boldsymbol{E}=\left[\boldsymbol{e}_{1}, \ldots \boldsymbol{e}_{\lambda N_{I_{i}}}\right] \in \mathbb{R}^{n_{\theta} \times \lambda N_{I_{i}}}$ are coefficient matrices to be determined. The matrices $\boldsymbol{T}, \boldsymbol{K}$ and $\boldsymbol{\Psi}$ represent the evaluations of the smooth, residual and edge components of the image intensity function on discrete grids. The superscript $\ell$ and $h$ denote the coarse and fine grids and correspond to lower and higher resolution, respectively. We obtain the fine and coarse scale matrices $\boldsymbol{T}$ and $\boldsymbol{K}$ following the discretization models outlined in section 2.1 Similarly, we follow the discretization in section 2.2 to generate $\boldsymbol{\Psi}^{\ell}$ and $\boldsymbol{\Psi}^{h}$. The coefficient matrices are obtained by solving the following minimization problem for each patch indexed by $i$ :

$$
\begin{aligned}
\min _{\boldsymbol{D}, \boldsymbol{C}, \boldsymbol{E}}\left\{\frac{1}{2}\left\|\boldsymbol{T}^{\ell} \boldsymbol{D}+\boldsymbol{K}^{\ell} \boldsymbol{C}+\boldsymbol{\Psi}^{\ell} \boldsymbol{E}-\boldsymbol{Y}_{\left(\lambda, I_{i}\right)}\right\|_{F}^{2}\right. \\
\left.+\frac{\mu_{1}}{2} \sum_{j k} w_{j k}\left\|\boldsymbol{d}_{j}-\boldsymbol{d}_{k}\right\|_{2}^{2}+\frac{\mu_{2}}{2} \operatorname{tr}\left(\boldsymbol{C}^{T} \boldsymbol{K}^{\ell} \boldsymbol{C}\right)+\mu_{3}\|\boldsymbol{E}\|_{1,1}\right\}
\end{aligned}
$$

with $\operatorname{tr}(\cdot)$ as the trace and adaptive weights

$$
w_{j k}=\exp \left(-\frac{\left\|\boldsymbol{d}_{j}-\boldsymbol{d}_{k}\right\|_{2}^{2}}{\sigma}\right), \quad \sigma>0,
$$

where $\boldsymbol{D}=\left[\boldsymbol{d}_{1}, \ldots \boldsymbol{d}_{\lambda N_{I_{i}}}\right]$ and $\mu_{1}, \mu_{2}, \mu_{3} \geq 0$. Note that $\exp \left(-\frac{\left\|\boldsymbol{d}_{j}-\boldsymbol{d}_{k}\right\|_{2}^{2}}{\sigma}\right)\left\|\boldsymbol{d}_{j}-\boldsymbol{d}_{k}\right\|_{2}^{2}$ is a unimodal function of $\left\|\boldsymbol{d}_{j}-\boldsymbol{d}_{k}\right\|_{2}^{2}$

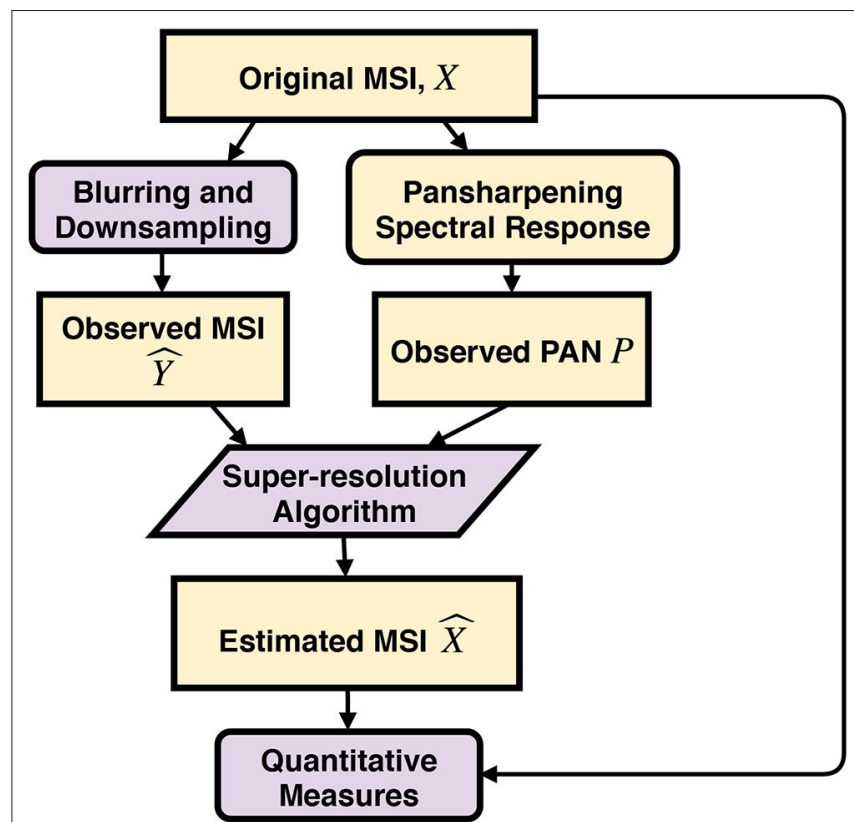

FIGURE 3 | Flow diagram of the experimental procedure for synthetic datasets.

that is maximized at 1 and minimized at zero and $\infty$. To simplify the computations, the adaptive weights are computed using the coefficients from a previous iteration. The first term of (26) is the data fidelity. There are three regularization terms in our proposed model. We assume that coefficients $\boldsymbol{d}_{i}$ are likely to vary smoothly within the same class of images. When $\boldsymbol{d}_{i}$ and $\boldsymbol{d}_{j}$ fall into the same class, $w_{i j}$ tends to be larger, forcing the next iterate of $\boldsymbol{d}_{i}$ and $\boldsymbol{d}_{j}$ to be close as well. The second regularization term is a structure guided regularity following from (16) to guarantee that the coefficients of the residual components do not grow too large. Finally, we impose sparsity constraints using the $\|\cdot\|_{1,1}$ norm [48] defined by

$$
\|\boldsymbol{A}\|_{1,1}=\sum_{s}^{S}\left\|\boldsymbol{a}_{s}\right\|_{1}, \boldsymbol{A}=\left[\boldsymbol{a}_{1}, \ldots, \boldsymbol{a}_{S}\right]
$$

since we assume that edges are sparse in the image patches. The resulting minimization can be solved using splitting techniques to obtain closed form solutions for the coefficients. We solve (26) iteratively using the alternating direction method of multipliers (ADMM) algorithm [49, 50].

Due to the non-differentiability of the $\|\cdot\|_{1,1}$ norm, we introduce a new variable $U$ and solve the equality-constrained optimization problem

$$
\begin{aligned}
& \min _{\boldsymbol{D}, \boldsymbol{C}, \boldsymbol{E}, \boldsymbol{U}}\left\{\frac{1}{2}\left\|\boldsymbol{T}^{\ell} \boldsymbol{D}+\boldsymbol{K}^{\ell} \boldsymbol{C}+\boldsymbol{\Psi}^{\ell} \boldsymbol{E}-\boldsymbol{Y}_{\left(\lambda, I_{i}\right)}\right\|_{F}^{2}\right. \\
& \left.+\frac{\mu_{1}}{2} \sum_{j k} w_{j k}\left\|\boldsymbol{d}_{j}-\boldsymbol{d}_{k}\right\|_{2}^{2}+\frac{\mu_{2}}{2} \operatorname{tr}\left(\boldsymbol{C}^{T} \boldsymbol{K}^{\ell} \boldsymbol{C}\right)+\mu_{3}\|\boldsymbol{U}\|_{1,1}\right\}
\end{aligned}
$$

subject to $\boldsymbol{U}=\boldsymbol{E}$. 


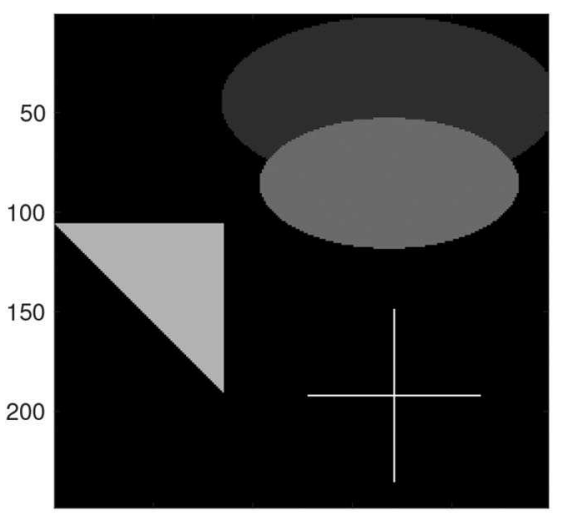

50

100

150

200

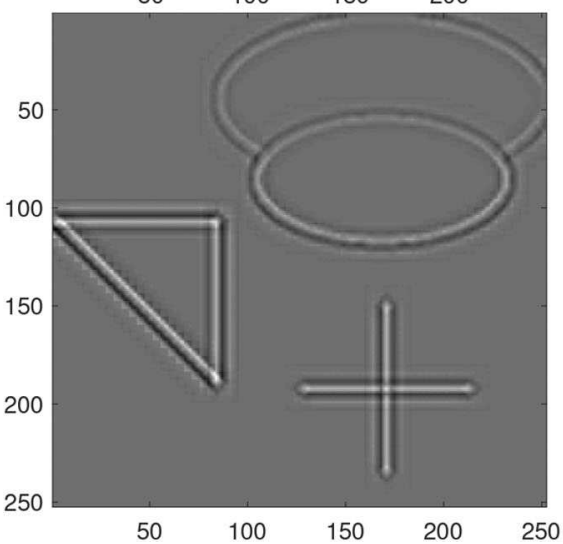

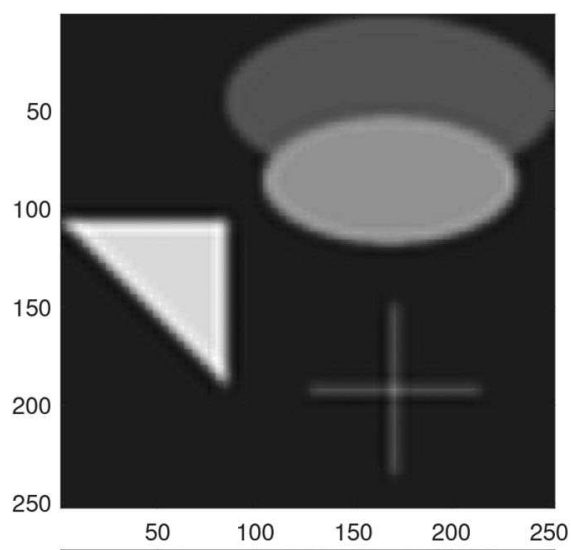

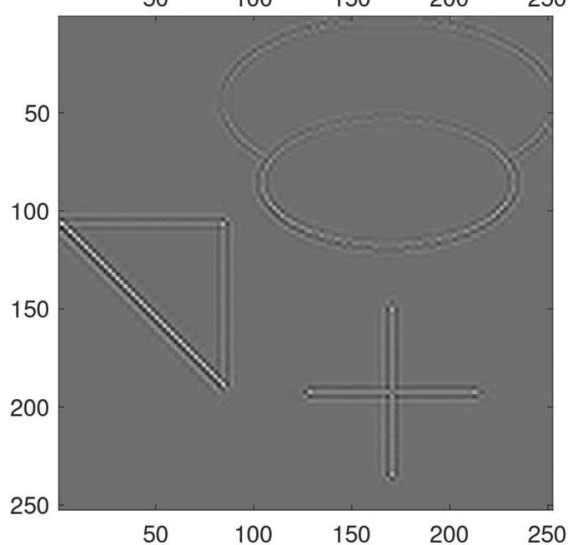

FIGURE 4 | Decomposition of components for the grayscale test image. From top to bottom and left to right: the original, $\boldsymbol{T}^{h} \boldsymbol{D}, \boldsymbol{K}^{h} \boldsymbol{C}, \boldsymbol{\Psi}^{h} \boldsymbol{E}$.

By introducing the augmented Lagrangian and completing the squares, we change the constrained optimization problem (29) into the following non-constrained optimization where $V$ is Lagrangian multiplier,

$$
\begin{aligned}
& \min _{\boldsymbol{D}, \boldsymbol{C}, \boldsymbol{E}, \boldsymbol{U}}\left\{\frac{1}{2}\left\|\boldsymbol{T}^{\ell} \boldsymbol{D}+\boldsymbol{K}^{\ell} \boldsymbol{C}+\boldsymbol{\Psi}^{\ell} \boldsymbol{E}-\boldsymbol{Y}_{\left(\lambda, I_{i}\right)}\right\|_{F}^{2}\right. \\
& +\frac{\mu_{1}}{2} \sum_{j k} w_{j k}\left\|\boldsymbol{d}_{j}-\boldsymbol{d}_{k}\right\|_{2}^{2}+\frac{\mu_{2}}{2} \operatorname{tr}\left(\boldsymbol{C}^{T} \boldsymbol{K}^{\ell} \boldsymbol{C}\right) \\
& \left.+\mu_{3}\|\boldsymbol{U}\|_{1,1}+\frac{\gamma}{2}\left\|\boldsymbol{U}-\boldsymbol{E}+\frac{\boldsymbol{V}}{\gamma}\right\|_{F}^{2}\right\},
\end{aligned}
$$

with step length $\gamma \geq 0$. ADMM reduces (30) to solving the following separable subproblems:

- $D$ subproblem:

$$
\begin{gathered}
\min _{\boldsymbol{D}}\left\{\frac{1}{2}\left\|\boldsymbol{T}^{\ell} \boldsymbol{D}+\boldsymbol{K}^{\ell} \boldsymbol{C}+\boldsymbol{\Psi}^{\ell} \boldsymbol{E}-\boldsymbol{Y}_{\left(\lambda, I_{i}\right)}\right\|_{F}^{2}\right. \\
\left.+\frac{\mu_{1}}{2} \sum_{j k} w_{j k}\left\|\boldsymbol{d}_{j}-\boldsymbol{d}_{k}\right\|_{2}^{2}\right\} .
\end{gathered}
$$

Given that $\boldsymbol{D}=\left[\boldsymbol{d}_{1}, \ldots \boldsymbol{d}_{\lambda N_{I_{i}}}\right], \boldsymbol{C}=\left[\boldsymbol{c}_{1}, \ldots \boldsymbol{c}_{\lambda N_{I_{i}}}\right], \boldsymbol{E}=$ $\left[\boldsymbol{e}_{1}, \ldots \boldsymbol{e}_{\lambda N_{I_{i}}}\right]$ and we can solve for each column of $\boldsymbol{D}$ separately $\min _{\boldsymbol{d}_{j}}\left\{\frac{1}{2}\left\|\boldsymbol{T}^{\ell} \boldsymbol{d}_{j}+\boldsymbol{K}^{\ell} \boldsymbol{c}_{j}+\boldsymbol{\Psi}^{\ell} \boldsymbol{e}_{j}-\boldsymbol{y}\right\|_{F}^{2}+\frac{\mu_{1}}{2} \sum_{j k} w_{j k}\left\|\boldsymbol{d}_{j}-\boldsymbol{d}_{k}\right\|_{2}^{2}\right\}$

where $y$ is the corresponding column in $\boldsymbol{Y}_{\left(\lambda, I_{i}\right)}$ matching the column index of $\boldsymbol{d}_{j}$. This gives a solution

$$
\begin{aligned}
\boldsymbol{d}_{j}= & \left(\boldsymbol{T}^{\ell T} \boldsymbol{T}^{\ell}+\mu_{1} \sum_{j k} w_{j k} \boldsymbol{I}\right)^{-1}\left(\boldsymbol{T}^{\ell T} \boldsymbol{T}^{\ell} \boldsymbol{y}-\boldsymbol{T}^{\ell T} \boldsymbol{K}^{\ell} \boldsymbol{c}_{j}\right. \\
& \left.-\boldsymbol{T}^{\ell T} \boldsymbol{\Psi}^{\ell} \boldsymbol{e}_{j}+\mu_{1} \sum_{j k} w_{j k} \boldsymbol{d}_{k}\right) .
\end{aligned}
$$

where $\boldsymbol{T}^{\ell_{T}}$ represents the transpose of $\boldsymbol{T}^{\ell}$.

- $C$ subproblem:

$$
\min _{\boldsymbol{C}}\left\{\frac{1}{2}\left\|\boldsymbol{T}^{\ell} \boldsymbol{D}+\boldsymbol{K}^{\ell} \boldsymbol{C}+\boldsymbol{\Psi}^{\ell} \boldsymbol{E}-\boldsymbol{Y}_{\left(\lambda, I_{i}\right)}\right\|_{F}^{2}+\frac{\mu_{2}}{2} \operatorname{tr}\left(\boldsymbol{C}^{T} \boldsymbol{K}^{\ell} \boldsymbol{C}\right)\right\} .
$$

From which we get

$$
\begin{aligned}
\boldsymbol{C}= & \left(\boldsymbol{K}^{\ell T} \boldsymbol{K}^{\ell}+\mu_{2} \boldsymbol{K}^{\ell}\right)^{-1}\left(\boldsymbol{K}^{\ell T} \boldsymbol{Y}_{\left(\lambda, I_{i}\right)}\right. \\
& \left.-\boldsymbol{K}^{\ell T} \boldsymbol{T}^{\ell} \boldsymbol{D}-\boldsymbol{K}^{\ell T} \boldsymbol{\Psi}^{\ell} \boldsymbol{E}\right) .
\end{aligned}
$$




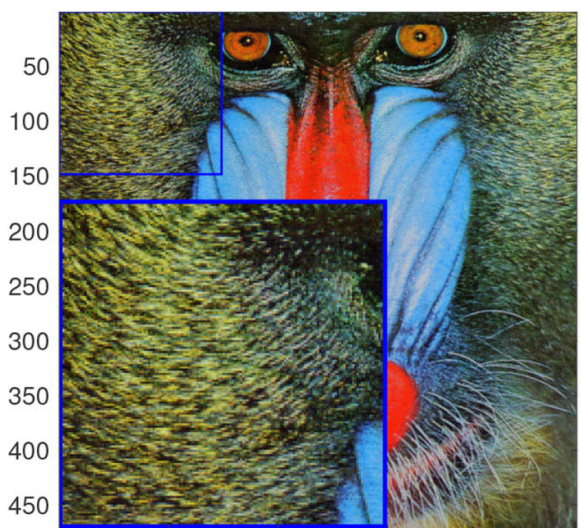

$\begin{array}{llll}100 & 200 & 300 & 400\end{array}$

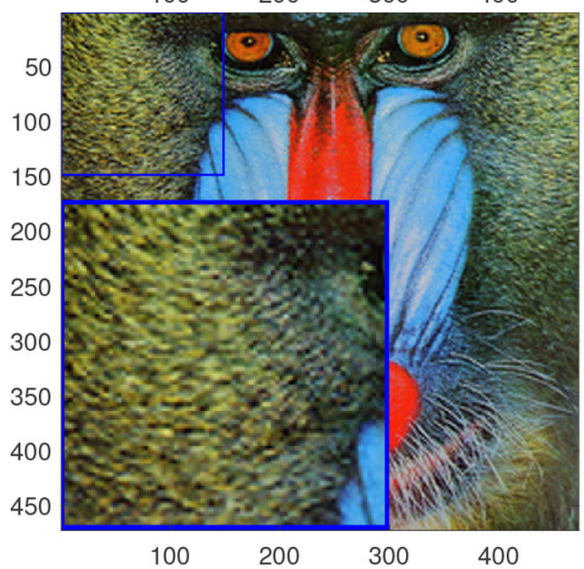

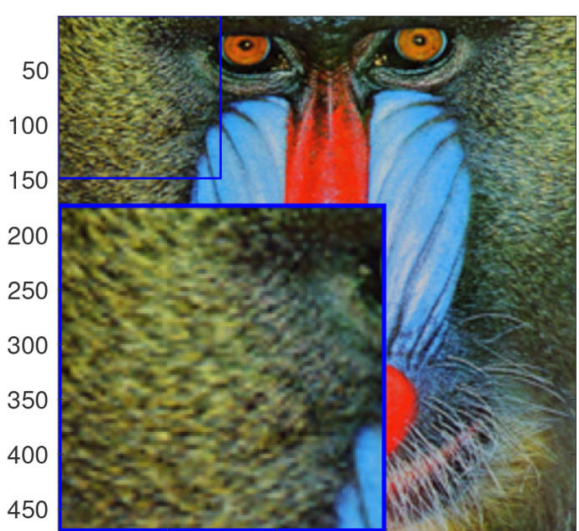

$\begin{array}{llll}100 & 200 & 300 & 400\end{array}$

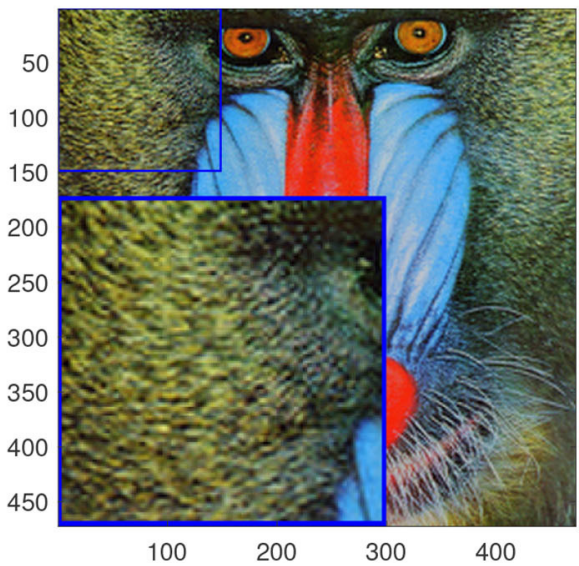

FIGURE 5 | Results for baboon using a scale factor $\omega=2$. From top to bottom and left to right: ground truth, bicubic interpolation, RKHS [40] and the proposed.

- $E$ subproblem:

$\min _{\boldsymbol{E}}\left\{\frac{1}{2}\left\|\boldsymbol{T}^{\ell} \boldsymbol{D}+\boldsymbol{K}^{\ell} \boldsymbol{C}+\boldsymbol{\Psi}^{\ell} \boldsymbol{E}-\boldsymbol{Y}_{\left(\lambda, I_{i}\right)}\right\|_{F}^{2}+\frac{\gamma}{2}\left\|\boldsymbol{U}-\boldsymbol{E}+\frac{\boldsymbol{V}}{\gamma}\right\|_{F}^{2}\right\}$.

Similarly, this gives the solution

$$
\begin{aligned}
\boldsymbol{E}= & \left(\boldsymbol{\Psi}^{\ell T} \boldsymbol{\Psi}^{\ell}+\gamma_{1} \boldsymbol{I}\right)^{-1}\left(\boldsymbol{\Psi}^{\ell T} \boldsymbol{Y}_{\left(\lambda, I_{i}\right)}-\boldsymbol{\Psi}^{\ell T} \boldsymbol{T}^{\ell} \boldsymbol{D}\right. \\
& \left.-\boldsymbol{\Psi}^{\ell T} \boldsymbol{K}^{\ell} \boldsymbol{C}+\gamma(\boldsymbol{U}+\boldsymbol{V})\right) .
\end{aligned}
$$

- $U$ subproblem:

$$
\min _{\boldsymbol{U}}\left\{\mu_{3}\|\boldsymbol{U}\|_{1,1}+\frac{\gamma}{2}\left\|\boldsymbol{U}-\boldsymbol{E}+\frac{\boldsymbol{V}}{\gamma}\right\|_{F}^{2}\right\} .
$$

A solution to this problem arising from the group sparsity constraint can be obtained by applying $\ell_{1}$ shrinkage to the rows of $\boldsymbol{U}$, i.e.,

$$
\boldsymbol{u}_{j}^{T}=\operatorname{shrink}\left(\boldsymbol{u}_{j}^{T}+\boldsymbol{v}_{j}^{T} / \gamma, \mu_{3} / \gamma\right)
$$

where

$$
\operatorname{shrink}(x, \gamma)= \begin{cases}x_{i}-v, & \text { if } x_{i}>\gamma \\ x_{i}+v, & \text { if } x_{i}<-\gamma \\ 0, & \text { otherwise }\end{cases}
$$

$$
\text { for any } \boldsymbol{x} \in \mathbb{R}^{n} \text { and } \gamma>0 .
$$

The update for $\boldsymbol{V}$ is obtained by

$$
\boldsymbol{V}=\boldsymbol{V}+\gamma(\boldsymbol{E}-\boldsymbol{U}) .
$$

The estimated set of patches $\mathscr{P}_{\widehat{X}}$ can be generated using (25) once the coefficients have been obtained. After running through the procedure for all the $k$ sets, we consolidate the patches to form the enhanced resolution result $\widehat{X}$. The proposed algorithm for SISR is summarized in Algorithm 1. Step 3 contains most of the key components of the algorithm so we provide more details. An outer layer loop is used to pick up residuals and put them back into the super resolution algorithm to further enhance the results. $\tau$ is the total number of outer layer iterations, $\boldsymbol{B}$ and $\boldsymbol{S}$ are the blurring and downsampling matrices, respectively. In the absence of blur, $\boldsymbol{B}$ becomes the identity matrix. Starting from one cluster of low resolution image patches, Step 3(b) solves the minimization problem (30) to obtain the coefficients $\boldsymbol{C}, \boldsymbol{D}, \boldsymbol{E}$, from which one is able to assemble a higher resolution image as shown in Step 3(c). From this higher resolution image, we can create a lower resolution image by applying $\boldsymbol{S B}$ operator on it. Step 3(d) calculates the error between the actual low resolution and the simulated one. If the higher resolution image is close to the ground truth, we expect the error to be small. Otherwise, 
we feeding this difference into the minimization problem (30) can further recover more details. These details are added to the previously recovered higher resolution image to achieve a better image [40].

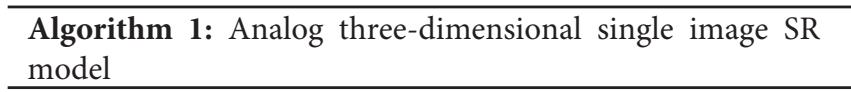

Input: $\boldsymbol{Y} \in \mathbb{R}^{m_{1} \times m_{2} \times \lambda}, \mu_{1}, \mu_{2}, \mu_{3}, \tau>0, \gamma, \sigma$

Output: $\widehat{\boldsymbol{X}} \in \mathbb{R}^{n_{1} \times n_{2} \times \lambda}$

Step 1. Construct the coarse and fine scale matrices $T, K$, and $\boldsymbol{\Psi}$ for the patches

Step 2. Decompose $\boldsymbol{Y}$ into sets of overlapping patches and cluster them into $k$ classes $\mathscr{P}_{Y}^{1}, \ldots, \mathscr{P}_{Y}^{k}$ using $k$-means clustering.

Step 3. Initialization for each class: $\boldsymbol{Y}_{\left(\lambda, I_{i}\right)}^{(1)} \leftarrow \boldsymbol{Y}_{\lambda, I_{i}}$

$$
\begin{aligned}
& \text { for } r \leftarrow 1 \text { to } \tau \text { do } \\
& \text { a. Initialize } \boldsymbol{C}^{(1)}, \boldsymbol{D}^{(1)}, \boldsymbol{E}^{(1)} \text { and } \boldsymbol{U}^{(1)} \text {. } \\
& \text { b. while not converged, } t=1, \ldots \text { do } \\
& \boldsymbol{D}^{(t+1)}=\underset{D}{\arg \min }\left\{\frac{1}{2} \| \boldsymbol{T}^{\ell} \boldsymbol{D}+\boldsymbol{K}^{\ell} \boldsymbol{C}^{(t)}+\boldsymbol{\Psi}^{\ell} \boldsymbol{E}^{(t)}-\right. \\
& \left.\boldsymbol{Y}_{\left(\lambda, I_{i}\right)}^{(r)}\left\|_{F}^{2}+\frac{\mu_{1}}{2} \sum_{j k} w_{j k}\right\| \boldsymbol{d}_{j}-\boldsymbol{d}_{k} \|_{2}^{2}\right\} \\
& \boldsymbol{C}^{(t+1)}=\underset{C}{\arg \min }\left\{\frac{1}{2} \| \boldsymbol{T}^{\ell} \boldsymbol{D}^{(t+1)}+\boldsymbol{K}^{\ell} \boldsymbol{C}+\boldsymbol{\Psi}^{\ell} \boldsymbol{E}^{(t)}-\right. \\
& \left.\boldsymbol{Y}_{\left(\lambda, I_{i}\right)}^{(r)} \|_{F}^{2}+\frac{\mu_{2}}{2} \operatorname{tr}\left(\boldsymbol{C}^{T} \boldsymbol{K}^{\ell} \boldsymbol{C}\right)\right\} \\
& \boldsymbol{E}^{(t+1)}=\underset{\boldsymbol{E}}{\arg \min }\left\{\frac{1}{2} \| \boldsymbol{T}^{\ell} \boldsymbol{D}^{(t+1)}+\boldsymbol{K}^{\ell} \boldsymbol{C}^{(t+1)}+\boldsymbol{\Psi}^{\ell} \boldsymbol{E}-\right. \\
& \left.\boldsymbol{Y}_{\left(\lambda, I_{i}\right)}^{(r)}\left\|_{F}^{2}+\frac{\gamma}{2}\right\| \boldsymbol{U}^{(t)}-\boldsymbol{E}+\frac{\boldsymbol{V}^{(t)}}{\gamma} \|_{F}^{2}\right\} \\
& \boldsymbol{U}^{(t+1)}=\underset{\boldsymbol{U}}{\arg \min }\left\{\mu_{3}\|\boldsymbol{U}\|_{1,1}+\frac{\gamma}{2} \| \boldsymbol{U}-\boldsymbol{E}^{(t+1)}+\right. \\
& \left.\frac{V^{(t)}}{\gamma} \|_{F}^{2}\right\} \\
& \boldsymbol{V}^{(t+1)} \leftarrow \boldsymbol{V}^{(t)}+\gamma\left(\boldsymbol{E}^{(t+1)}-\boldsymbol{U}^{(t+1)}\right) \\
& \text { c. } \widehat{\boldsymbol{X}}_{\left(\lambda, I_{i}\right)}^{(r)} \leftarrow \boldsymbol{T}^{h} \boldsymbol{D}^{(t+1)}+\boldsymbol{K}^{h} \boldsymbol{C}^{(t+1)}+\boldsymbol{\Psi}^{h} \boldsymbol{E}^{(t+1)} \\
& \text { d. } \boldsymbol{Y}_{\left(\lambda, I_{i}\right)}^{(r+1)} \leftarrow \boldsymbol{Y}_{\left(\lambda, I_{i}\right)}^{(r)}-\boldsymbol{S B}\left(\widehat{\boldsymbol{X}}_{\left(\lambda, I_{i}\right)}^{(r)}\right)
\end{aligned}
$$

Step 4. $\widehat{\boldsymbol{X}}_{\left(\lambda, I_{i}\right)}=\sum_{t=1}^{\tau} \widehat{\boldsymbol{X}}_{\left(\lambda, I_{i}\right)}^{(r)}$

Step 5. Consolidate the patches $\mathscr{P}_{\widehat{X}}^{1}, \ldots, \mathscr{P}_{\widehat{X}}^{k}$ to obtain $\widehat{X}$.

\subsection{Pansharpening}

Given a LR MS image $\boldsymbol{Y}$ and a high spatial resolution PAN image $(\boldsymbol{P})$ matching the same scene, our goal is to estimate a HR MS image $\widehat{\boldsymbol{X}}$ with the spatial dimensions of the PAN. We propose a two-stage approach to solve the pansharpening problem. To begin with, we obtain a preliminary estimate for the target image $\widetilde{\boldsymbol{Y}}$ using CS or MRA, two fast and simple pansharpening techniques. $\widetilde{\boldsymbol{Y}}$ has the same size as $\widehat{\boldsymbol{X}}$ but the result $\widetilde{\boldsymbol{Y}}$ obtained using these methods suffer from spectral and spatial distortion drawbacks. The second step in our proposed twostage approach provides a way to reduce the drawbacks. Next, we feed Algorithm 1 with input $\widetilde{Y}$ and proceed with the resulting optimization model to generate $\widehat{\boldsymbol{X}}$. Considering the image $\widetilde{\boldsymbol{Y}}$, the modified optimization model becomes

$$
\begin{aligned}
\min _{\boldsymbol{D}, \boldsymbol{C}, \boldsymbol{E}}\left\{\frac{1}{2}\left\|\boldsymbol{T}^{h} \boldsymbol{D}+\boldsymbol{K}^{h} \boldsymbol{C}+\boldsymbol{\Psi}^{h} \boldsymbol{E}-\widetilde{\boldsymbol{Y}}_{\left(\lambda, I_{i}\right)}\right\|_{F}^{2}\right. \\
\left.+\frac{\mu_{1}}{2} \sum_{j k} w_{j k}\left\|\boldsymbol{d}_{j}-\boldsymbol{d}_{k}\right\|_{2}^{2}+\frac{\mu_{2}}{2} \operatorname{tr}\left(\boldsymbol{C}^{T} \boldsymbol{K}^{\ell} \boldsymbol{C}\right)+\mu_{3}\|\boldsymbol{E}\|_{1,1}\right\},
\end{aligned}
$$

where $\widetilde{\boldsymbol{Y}}_{\left(\lambda, I_{i}\right)}=\left[\widetilde{\boldsymbol{y}}_{1, p_{1}}, \ldots, \tilde{\boldsymbol{y}}_{\lambda, p_{1}}, \ldots, \widetilde{\boldsymbol{y}}_{1, p_{N_{I_{i}}}}, \ldots, \widetilde{\boldsymbol{y}}_{\lambda, p_{N_{I_{i}}}}\right] \in$ $\mathbb{R}^{n_{p} n_{p} \times \lambda N_{I_{i}}}$ for the set of patches $\mathscr{P}_{\widetilde{Y}}^{i}, 1 \leq i \leq k$. K-means clustering is used to decompose the number of overlapping patches into $k$ classes, so that pixels corresponding to similar kinds of objects in the image will be collectively estimated in each step. The optimal value of $k$ for a given image was determined using the silhouette method [51] which measures how similar a point is to its own cluster compared to other clusters. The adaptive weights are defined the same way as in (27). Applying $\mathrm{ADMM}$ to (31) we can solve the separable subproblems that ensue for the coefficients $\boldsymbol{D}, \boldsymbol{C}$ and $\boldsymbol{E}$ as in the single image model. We summarize the procedure in Algorithm 2.

\section{EXPERIMENTAL RESULTS}

\subsection{Simulation Protocol}

To begin with the simulation procedure, we test the performance of the proposed algorithm by generating the reduced resolution image from a given image for some chosen downscaling factors. We then compute the enhanced resolution result using the proposed algorithm and compare its performance with other methods. The experiments are implemented using MATLAB(R2018a).

For the SISR experiments we compare our three-dimensional single image super-resolution algorithm to bicubic interpolation and the RKHS algorithm by Deng et al. [40]. The authors in Deng et al. [40] implement their SR algorithm following an independent band procedure. For color images (RGB), they transform the image to the "YCbCr" color space and apply model in Deng et al. [40] to the luma component (Y) only since humans are more sensitive to luminance changes. The " $\mathrm{Cb}$ " and "Cr" components are the blue-difference and the red-difference chroma components, respectively. This way, the time complexity of the algorithm is reduced, as compared to an optimization based methods using all the channels at the same time. To obtain the desired result, the chroma components $(\mathrm{Cb}, \mathrm{Cr})$ are upscaled by bicubic interpolation. The components are then transformed back to the RGB color space for further analysis. In our approach, we apply Algorithm 1 to the three-dimensional color images directly, without undertaking any transformations. 
We undertake the pansharpening experiments using semisynthetic test data. This may be attributed to the fact that existing sensors cannot readily provide images at the increased spatial resolution that is desired. A generally acceptable procedure attributed to Ranchin and Wald [23] for operating at reduced resolution is as follows (Illustration in Figure 3):

- The enhanced result should be as identical as possible to the original multispectral image once it has been degraded.

- The enhanced result should be identical to the corresponding high resolution image that a capable sensor would observe at the increased resolution.

- Each band of the enhance result should be as identical as possible to the corresponding bands of the image

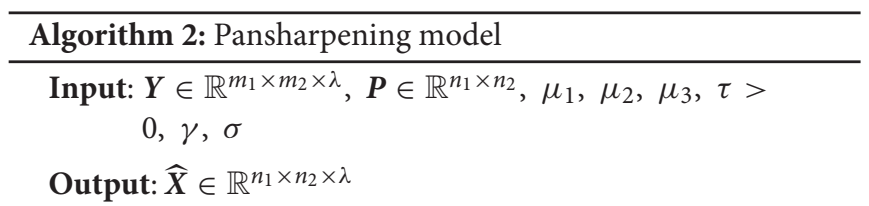

Step 1. Construct the coarse and fine scale matrices $T, K$, and $\boldsymbol{\Psi}$ for the patches.

Step 2. Obtain the upscaled image $\widetilde{\boldsymbol{Y}}$ using $\boldsymbol{P}$.

Step 3. Decompose $\tilde{\boldsymbol{Y}}$ into overlapping patches and cluster them into $k$ classes $\mathscr{P}_{\widetilde{Y}}^{1}, \ldots, \mathscr{P}_{\widetilde{Y}}^{k}$ using $k$-means clustering.

Step 4. Initialization for each class: $\tilde{Y}_{\left(\lambda, I_{i}\right)}^{(1)} \leftarrow \widetilde{Y}_{\lambda, I_{i}}$

$$
\begin{aligned}
& \text { for } r \leftarrow 1 \text { to } \tau \text { do } \\
& \text { a. Initialize } \boldsymbol{C}^{(1)}, \boldsymbol{D}^{(1)} \text { and } \boldsymbol{E}^{(1)} \text {. } \\
& \text { b. while not converged, } t=1, \cdots \text { do } \\
& \boldsymbol{D}^{(t+1)}=\underset{\boldsymbol{D}}{\arg \min }\left\{\frac{1}{2} \| \boldsymbol{T}^{h} \boldsymbol{D}+\boldsymbol{K}^{h} \boldsymbol{C}^{(t)}+\boldsymbol{\Psi}^{h} \boldsymbol{E}^{(t)}-\right. \\
& \left.\widetilde{\boldsymbol{Y}}_{\left(\lambda, I_{i}\right)}^{(r)}\left\|_{F}^{2}+\frac{\mu_{1}}{2} \sum_{j k} w_{j k}\right\| \boldsymbol{d}_{j}-\boldsymbol{d}_{k} \|_{2}^{2}\right\} \\
& \boldsymbol{C}^{(t+1)}=\underset{\boldsymbol{C}}{\arg \min }\left\{\frac{1}{2} \| \boldsymbol{T}^{h} \boldsymbol{D}^{(t+1)}+\boldsymbol{K}^{h} \boldsymbol{C}+\boldsymbol{\Psi}^{h} \boldsymbol{E}^{(t)}-\right. \\
& \left.\widetilde{\boldsymbol{Y}}_{\left(\lambda, I_{i}\right)}^{(r)} \|_{F}^{2}+\frac{\mu_{2}}{2} \operatorname{tr}\left(\boldsymbol{C}^{T} \boldsymbol{K}^{\ell} \boldsymbol{C}\right)\right\} \\
& \boldsymbol{E}^{(t+1)}=\underset{\boldsymbol{E}}{\arg \min }\left\{\frac{1}{2} \| \boldsymbol{T}^{h} \boldsymbol{D}^{(t+1)}+\boldsymbol{K}^{h} \boldsymbol{C}^{(t+1)}+\boldsymbol{\Psi}^{h} \boldsymbol{E}-\right. \\
& \left.\tilde{\boldsymbol{Y}}_{\left(\lambda, I_{i}\right)}^{(r)}\left\|_{F}^{2}+\frac{\gamma}{2}\right\| \boldsymbol{U}^{(t)}-\boldsymbol{E}+\frac{\boldsymbol{V}^{(t)}}{\gamma} \|_{F}^{2}\right\} \\
& \boldsymbol{U}^{(t+1)}=\underset{\boldsymbol{U}}{\arg \min }\left\{\mu_{3}\|\boldsymbol{U}\|_{1,1}+\frac{\gamma}{2}\left\|\boldsymbol{U}-\boldsymbol{E}^{(t+1)}+\frac{\boldsymbol{V}^{(t)}}{\gamma}\right\|_{F}^{2}\right\} \\
& \boldsymbol{V}^{(t+1)} \leftarrow \boldsymbol{V}^{(t)}+\gamma\left(\boldsymbol{E}^{(t+1)}-\boldsymbol{U}^{(t+1)}\right) \\
& \text { c. } \widehat{\boldsymbol{X}}_{\left(\lambda, I_{i}\right)}^{(r)} \leftarrow \boldsymbol{T}^{h} \boldsymbol{D}^{(t+1)}+\boldsymbol{K}^{h} \boldsymbol{C}^{(t+1)}+\boldsymbol{\Psi}^{h} \boldsymbol{E}^{(t+1)} \\
& \text { d. } \widetilde{\boldsymbol{Y}}_{\left(\lambda, I_{i}\right)}^{(t+1)} \leftarrow \widetilde{\boldsymbol{Y}}_{\left(\lambda, I_{i}\right)}^{(r)}-\widehat{\boldsymbol{X}}_{\left(\lambda, I_{i}\right)}^{(r)}
\end{aligned}
$$

Step 5. $\widehat{X}_{\left(\lambda, I_{i}\right)}=\sum_{t=1}^{\tau} \widehat{\boldsymbol{X}}_{\left(\lambda, I_{i}\right)}^{(r)}$

Step 6. Consolidate the patches $\mathscr{P}_{\widehat{X}}^{1}, \ldots, \mathscr{P}_{\widehat{X}}^{k}$ to obtain $\widehat{X}$. that would have been observed by a sensor with high resolution.

We apply Algorithm 2 to the observed multispectral data and compare it with existing state-of-the-art approaches.

\subsection{Parameter Selection}

Parameter selection is essential to obtain good results. However, to show the stability of our proposed method, we fix the parameters for all the experiments. Fine tuning of the parameters might lead to slightly better results. Parameter choices for the optimization of our proposed algorithms are outlined as follows:

- Scale factor $(\omega)$ : In the experiments shown above, we set $\omega=2$ for the SISR problem, and set $\omega=4$ for the pansharpening problem. Quantitative measures obtained suggest that for $\omega>$ 4 both problems yield unsatisfactory results as the spatial and spectral measures are less competitive. However, the choice of $\omega$ depends on the dimensions of the observed data. For large $\omega$, we can select larger patch sizes to improve upon the target.

- Patch size $\left(m_{p}\right)$ : Patch sizes can vary according to the size of the given image. We use a default patch size, $m_{p} \times m_{p} \times \lambda$ in our experiments. Reasonable quantitative measures were obtained for $6 \leq m_{p} \leq 8$ for the two problems considered. Outside the given bounds, the algorithms either take time to converge or yield unsatisfactory results.

- Overlap size: The limits of the overlapping region range from 2 to 4 are limited by the patch size. We set the overlap size to 2 .

- Number of classes $(k)$ : In our experiments, the value of $k$ was decided based on the solution that gave the best result from the silhouette method [51], i.e., by combing through predetermined values. Future work will involve investigating whether it is possible to automate this step.

\begin{tabular}{llrrrr}
\multicolumn{5}{l}{ TABLE 1 } & \multicolumn{4}{l}{ Quantitative Results with for single image super-resolution with $\omega=2}$. \\
\hline Image & Index & PSNR & RMSE & SSIM & SAM \\
\hline \multirow{4}{*}{ baboon } & Bicubic & 22.6453 & 0.0737 & 0.9700 & 4.9851 \\
& RKHS [40] & 22.8789 & 0.0718 & 0.9770 & 5.1105 \\
& Proposed & 23.1566 & 0.0695 & 0.9780 & 4.9757 \\
& Bicubic & 29.7291 & 0.0326 & 0.9453 & 1.3056 \\
girl & RKHS [40] & 29.9922 & 0.0317 & 0.9564 & 1.3559 \\
& Proposed & 30.7616 & 0.0290 & 0.9621 & 1.2173 \\
& Bicubic & 32.3586 & 0.0241 & 0.9740 & 1.5280 \\
lena & RKHS [40] & 32.6024 & 0.0234 & 0.9810 & 1.5230 \\
& Proposed & 33.2650 & 0.0217 & 0.9890 & 1.4663 \\
& Bicubic & 29.6166 & 0.0330 & 0.9765 & 1.3992 \\
lion & RKHS [40] & 30.1480 & 0.0311 & 0.9788 & 1.6617 \\
& Proposed & 31.2364 & 0.0274 & 0.9801 & 1.3770 \\
& Bicubic & 31.4943 & 0.0266 & 0.9807 & 2.2896 \\
& RKHS [40] & 31.6163 & 0.0263 & 0.9820 & 2.6242 \\
face & Proposed & 31.9927 & 0.0251 & 0.9829 & 2.4506 \\
& Reference & $\infty$ & 0 & 1 & 0
\end{tabular}


TABLE 2 | Quantitative Results with for pansharpening $\omega=4$.

\begin{tabular}{|c|c|c|c|c|c|c|}
\hline Image & Index & SAM & SCC & RMSE & ERGAS & PSNR \\
\hline & PCA & 5.01 & 0.93 & 38.19 & 4.40 & 30.14 \\
\hline & IHS & 5.29 & 0.93 & 35.72 & 4.17 & 30.26 \\
\hline \multirow[t]{11}{*}{ Pléiades } & Brovey & 4.66 & 0.94 & 35.35 & 4.07 & 30.55 \\
\hline & GS & 4.75 & 0.96 & 34.48 & 4.00 & 30.86 \\
\hline & Indusion & 4.52 & 0.92 & 37.47 & 4.36 & 30.11 \\
\hline & HPF & 3.64 & 0.96 & 27.48 & 3.23 & 32.52 \\
\hline & SFIM & 3.61 & 0.96 & 27.18 & 3.17 & 32.75 \\
\hline & AWLP & 3.29 & 0.96 & 23.63 & 2.84 & 33.61 \\
\hline & GLP & 3.23 & 0.97 & 22.45 & 2.61 & 34.42 \\
\hline & RKHS [43] & 3.19 & 0.97 & 23.32 & 2.58 & 34.15 \\
\hline & Proposed & 3.17 & 0.98 & 20.19 & 2.23 & 36.42 \\
\hline & PCA & 3.52 & 0.96 & 20.34 & 2.64 & 33.25 \\
\hline & IHS & 5.04 & 0.89 & 27.10 & 3.56 & 29.72 \\
\hline \multirow[t]{11}{*}{ China } & Brovey & 4.49 & 0.90 & 24.12 & 3.15 & 30.63 \\
\hline & GS & 3.51 & 0.96 & 20.82 & 2.76 & 32.70 \\
\hline & Indusion & 3.89 & 0.94 & 25.28 & 3.25 & 31.54 \\
\hline & HPF & 3.32 & 0.96 & 20.84 & 2.69 & 33.16 \\
\hline & SFIM & 3.27 & 0.96 & 20.60 & 2.65 & 33.27 \\
\hline & AWLP & 3.02 & 0.96 & 19.20 & 2.50 & 33.61 \\
\hline & GLP & 3.27 & 0.96 & 20.46 & 2.71 & 32.78 \\
\hline & RKHS [43] & 3.06 & 0.96 & 19.52 & 2.56 & 33.36 \\
\hline & Proposed & 2.96 & 0.96 & 18.38 & 2.48 & 33.53 \\
\hline & PCA & 4.83 & 0.90 & 28.39 & 4.75 & 36.82 \\
\hline & IHS & 4.96 & 0.87 & 28.73 & 4.78 & 36.36 \\
\hline \multirow[t]{11}{*}{ Toulouse } & Brovey & 5.23 & 0.85 & 29.62 & 4.91 & 36.00 \\
\hline & GS & 4.76 & 0.90 & 28.12 & 4.71 & 36.90 \\
\hline & Indusion & 4.54 & 0.83 & 29.41 & 4.88 & 36.33 \\
\hline & HPF & 3.99 & 0.91 & 23.13 & 3.89 & 38.65 \\
\hline & SFIM & 3.95 & 0.92 & 22.76 & 3.83 & 38.78 \\
\hline & AWLP & 4.31 & 0.92 & 22.73 & 3.85 & 39.03 \\
\hline & GLP & 3.76 & 0.93 & 21.05 & 3.54 & 39.46 \\
\hline & RKHS [43] & 3.65 & 0.92 & 21.47 & 3.58 & 39.54 \\
\hline & Proposed & 3.41 & 0.92 & 20.83 & 3.47 & 39.28 \\
\hline & PCA & 11.66 & 0.85 & 0.06 & 14.05 & 28.34 \\
\hline & IHS & 6.30 & 0.69 & 0.04 & 18.67 & 25.49 \\
\hline \multirow[t]{11}{*}{ Deimos } & Brovey & 3.64 & 0.77 & 0.05 & 16.46 & 25.88 \\
\hline & GS & 5.77 & 0.81 & 0.05 & 15.30 & 26.52 \\
\hline & Indusion & 4.64 & 0.88 & 0.04 & 12.70 & 28.23 \\
\hline & HPF & 4.53 & 0.89 & 0.04 & 12.30 & 28.52 \\
\hline & SFIM & 4.68 & 0.69 & 0.19 & 102.38 & 22.38 \\
\hline & AWLP & 8.39 & 0.86 & 0.06 & 15.04 & 27.57 \\
\hline & GLP & 4.73 & 0.88 & 0.04 & 12.74 & 28.21 \\
\hline & RKHS [43] & 3.94 & 0.96 & 0.03 & 3.44 & 33.20 \\
\hline & Proposed & 3.89 & 0.94 & 0.03 & 9.01 & 31.22 \\
\hline & PCA & 4.50 & 0.96 & 62.23 & 4.31 & 30.98 \\
\hline & IHS & 5.04 & 0.95 & 65.75 & 4.48 & 30.60 \\
\hline \multirow[t]{10}{*}{ W2 } & Brovey & 4.86 & 0.95 & 65.01 & 4.49 & 30.43 \\
\hline & GS & 4.52 & 0.96 & 62.52 & 4.33 & 30.94 \\
\hline & Indusion & 4.56 & 0.91 & 74.77 & 5.14 & 29.38 \\
\hline & HPF & 4.10 & 0.95 & 57.14 & 3.96 & 31.72 \\
\hline & SFIM & 4.14 & 0.95 & 56.80 & 3.94 & 31.78 \\
\hline & AWLP & 4.62 & 0.95 & 52.82 & 3.88 & 32.26 \\
\hline & GLP & 3.90 & 0.96 & 50.92 & 3.55 & 32.70 \\
\hline & RKHS [43] & 3.88 & 0.96 & 47.32 & 3.30 & 33.35 \\
\hline & Proposed & 3.84 & 0.96 & 47.19 & 3.32 & 33.38 \\
\hline & Reference & 0 & 1 & 0 & 0 & $\infty$ \\
\hline
\end{tabular}

The best and the second best results are in bold face and italic bold face.
- Construction of approximation matrices $T, K$, and $\Psi$ : We generate the matrices based on the procedure outlined in section 3, with $M=6, \varepsilon=1 e^{-3}$, and set 20 evenly distributed angles on $[0,2 \pi]$ in computing the fine and coarse scale matrices for $\boldsymbol{T}, \boldsymbol{K}$ and $\boldsymbol{\Psi}$, respectively.

- Regularization penalties $\mu_{1}, \mu_{2}, \mu_{3}$ and $\gamma$ : Without knowledge of optimal values for the penalty, we sweep through a range of values to determine the optimal result. For the experiments considered, $\mu_{1}, \mu_{2}, \mu_{3}, \gamma \in\left\{10^{-8}, \cdots, 10^{2}\right\}$ and choose the one that leads to the best results evaluated by quantitative and qualitative measures. The step length $\gamma$ for updating of $V$ influences the convergence of the algorithm greatly.

- Preprocessing for Algorithm 2: Our proposed pansharpening method provides a flexible way to improve the results for both CS and MRA algorithms. In the experiments shown above, we preprocess the images using the GS algorithm. Future work will detail which preprocessing step works best for multispectral images.

\subsection{Quantitative Criteria}

To measure the similarity of the enhanced resolution image $(\widehat{\boldsymbol{X}})$ to the reference image $(\boldsymbol{X})$, we compute the following image quality metrics:

- Correlation coefficient (CC): The correlation coefficient measures the similarity of spectral features between the reference and pansharpened image. It is defined as

$$
\left.\operatorname{CC}(\widehat{\boldsymbol{X}})=\frac{1}{\lambda} \sum_{i=1}^{\lambda} \frac{1}{\sigma_{\widehat{\boldsymbol{X}}_{i}} \sigma_{\boldsymbol{X}_{i}}} \mathrm{E}\left[\widehat{\boldsymbol{X}}_{i}-\mu_{\widehat{\boldsymbol{X}}_{i}}\right)\left(\boldsymbol{X}_{i}-\mu_{\boldsymbol{X}_{i}}\right)\right]
$$

where $\boldsymbol{X}_{i}$ and $\widehat{\boldsymbol{X}}_{i}$ are the matrices of the $i$-th band of $\boldsymbol{X}$ and $\widehat{\boldsymbol{X}}$, with $\mu_{\boldsymbol{X}_{i}}$ and $\mu_{\widehat{\boldsymbol{X}}_{i}}$ as their respective mean gray values. The standard deviations for $\widehat{\boldsymbol{X}}_{i}$ and $\boldsymbol{X}_{i}$ are $\sigma_{\widehat{\boldsymbol{X}}_{i}}$ and $\sigma_{\boldsymbol{X}_{i}}$, respectively.

- Root mean square error (RMSE):

$$
\operatorname{RMSE}(\widehat{\boldsymbol{X}})=\sqrt{\operatorname{MSE}(\widehat{\boldsymbol{X}})}
$$

where

$$
\operatorname{MSE}(\widehat{\boldsymbol{X}})=E\left[(\widehat{\boldsymbol{X}}-\boldsymbol{X})^{2}\right]=\frac{\|\widehat{\boldsymbol{X}}-\boldsymbol{X}\|_{F}^{2}}{n_{1} n_{2} \lambda}
$$

- Peak signal-to-noise ratio (PSNR): It is an expression of the ratio between the maximum possible value of the signal and the distorting noise that affects the quality of the representation.

$$
\operatorname{PSNR}(\widehat{\boldsymbol{X}})=20 \cdot \log _{10}\left(\frac{\operatorname{MAX}_{\widehat{\boldsymbol{X}}}}{\sqrt{\operatorname{MSE}(\widehat{\boldsymbol{X}})}}\right)
$$

- Structural Similarity (SSIM): The SSIM index is computed by examining various windows of the reference and target image. It is a full reference metric designed to improve upon the traditional methods such as PNSR and MSE. 
- Spectral angle mapper (SAM): This measure denotes the absolute value of the angle between the reference and estimated spectral vectors.

Let $\widehat{\boldsymbol{X}}_{\{i\}}=\left(\hat{x}_{i, 1}, \ldots, \hat{x}_{i, \lambda}\right)^{T}, 1 \leq i \leq n_{1} n_{2}$ be a vector collecting the intensity at all spectral bands at pixel $i$,

$$
\operatorname{SAM}(\widehat{\boldsymbol{X}})=\frac{1}{n_{1} n_{2}} \sum_{i=1}^{n_{1} n_{2}} \arccos \left(\frac{\left\langle\widehat{\boldsymbol{X}}_{\{i\}}, \boldsymbol{X}_{\{i\}}\right\rangle}{\left\|\widehat{\boldsymbol{X}}_{\{i\}}\right\|\left\|\boldsymbol{X}_{\{i\}}\right\|}\right)
$$

- Erreur relative globale adimensionnelle de synthése (ERGAS): The relative dimensionless global error in the synthesis reflects the overall quality of the pansharpened image.

$$
\begin{gathered}
\operatorname{ERGAS}(\widehat{\boldsymbol{X}})=100 d \sqrt{\frac{1}{\lambda} \sum_{i=1}^{\lambda}\left(\frac{\operatorname{RMSE}\left(\widehat{\boldsymbol{X}}_{i}\right)}{\mu_{\widehat{\boldsymbol{X}}_{i}}}\right)^{2}}, \\
d=\text { downsampling factor. }
\end{gathered}
$$

- $Q$ index: It defines a universal measure that combines the loss of correlation, luminance, and contrast distortion of an image and is defined by

$$
Q(\widehat{\boldsymbol{X}})=\frac{1}{\lambda} \sum_{i=1}^{\lambda} \frac{4 \sigma_{\left(\boldsymbol{X}_{i}, \widehat{\boldsymbol{X}}_{i}\right)} \mu_{\boldsymbol{X}_{i}} \mu_{\widehat{\boldsymbol{X}}_{i}}}{\left.\left(\sigma_{\boldsymbol{X}_{i}}^{2}+\sigma_{\widehat{\boldsymbol{X}}_{i}}^{2}\right)\left[\mu_{\boldsymbol{X}_{i}}^{2}+\mu_{\widehat{\boldsymbol{X}}_{i}}^{2}\right]\right)}
$$

where $\sigma_{\left(\boldsymbol{X}_{i}, \widehat{\boldsymbol{X}}_{i}\right)}$ is the covariance between $\boldsymbol{X}_{i}$ and $\widehat{\boldsymbol{X}}_{i}$.

\subsection{Results}

In this section, we compare the visual results and quantitative measures of the proposed approaches with some existing superresolution algorithms. The results of Algorithm 1 will be compared to bicubic interpolation and the RKHS model by Deng et al. [40]. Results of Algorithm 2 are compared with CS methods such as PCA, IHS, Brovey, GS and Indusion, as well as MRA methods such as HPF, SFIM, ATWT, AWLP, GLP techniques, and the Deng et al. pansharpening model [43]. We begin with numerical tests for true color images and consider patches of size $8 \times 8 \times \lambda$. The size of the overlap across the vertical and horizontal dimensions is 2 .

In Figure 4, we show one example of decomposing an original image into three components. One can see that the edge information is separated successfully from the smooth component and the residual. In Figure 5, we compare 3D super resolution result of the proposed model with bicubic interpolation and RKHS [40] on a baboon image. We can see that the proposed method leads to a color image with highest visual quality. The bicubic interpolation result tends to be blurry. RKHS [40] result is better but not as good as the proposed. In Table 1, we

\section{REFERENCES}

1. Farsiu S, Robinson D, Elad M, Milanfar P. Advances and challenges in super-resolution. Int J Imaging Syst Technol. (2004) 14:47-57. doi: 10.1002/ima.20007 list some quantitative metric comparison for more testing images. It is observed that the proposed $3 \mathrm{D}$ single image super resolution method (detailed in Algorithm 1) is consistently the best among the three approaches.

For pansharpening, we compare with 10 other methods in the literature using five different quantitative metrics (see Table 2). It is observed that the proposed pansharpening method (detailed in Algorithm 2) is mostly the best and occasionally the second among all. The performance is consistently superior.

Note that we did not conduct comparison with deep neural network based methods. The proposed approach is based on single image modeling while the deep neural network approach relies on a lot of external data. We don't think it is a fair comparison.

\section{CONCLUSIONS}

In this paper, we have proposed a technique for single image SR by modeling the image as a linear combination of regular functions in tandem with sparse coding. We show that the proposed scheme is an improvement upon similar existing approaches as it outperforms these algorithms. Besides this advantage, the proposed methods can also benefit image decomposition.

In future work, we will apply the model to multispectral and hyperspectral image SR where sparse coding can be useful due to the redundant nature of the input.

\section{DATA AVAILABILITY STATEMENT}

Some of the datasets for this study can be found in http:// openremotesensing.net.

\section{AUTHOR CONTRIBUTIONS}

WG contributes on providing the main ideas including the mathematical models and algorithms proposed in the paper. RL was a former Ph.D. student of WG. Under WG's guidance, $\mathrm{RL}$ did computer programming to implement the ideas and conducted numerical experiments to test the effective of the proposed method compared to the state of the art. XZ and CG are domain scientists who help us evaluate the numerical results and provide comments.

\section{FUNDING}

WG was supported by National Science Foundation (DMS-1521582). 
Mag.

3207

4. Baker S, Kanade T. Limits on super-resolution and how to break them. IEEE Trans Pattern Anal Mach Intell. (2002) 24:1167-83. doi: 10.1109/TPAMI.2002.1033210

5. Lin Z, Shum H-Y. Fundamental limits of reconstruction-based superresolution algorithms under local translation. IEEE Trans Pattern Anal Mach Intell. (2004) 26:83-97. doi: 10.1109/TPAMI.2004.12 61081

6. Thévenaz $\mathrm{P}$, Blu T, Unser $\mathrm{M}$. Interpolation revisited [medical images application]. IEEE Trans Pattern Anal Mach Intell. (2000) 19:739-758. doi: $10.1109 / 42.875199$

7. Keys R. Cubic convolution interpolation for digital image processing. IEEE Trans Acoust Speech Signal Process. (1981) 29:1153-60. doi: 10.1109/TASSP.1981.1163711

8. Glasner D, Bagon S, Irani M. Super-resolution from a single image. In: IEEE 12th International Conference on Computer Vision, 2009. IEEE (2009). p. 349-56. doi: 10.1109/ICCV.2009.5459271

9. Freedman G, Fattal R. Image and video upscaling from local selfexamples. ACM Trans Graph. (2011) 30:12. doi: 10.1145/2010324.1964943

10. Gao X, Zhang K, Tao D, Li X. Joint learning for single-image super-resolution via a coupled constraint. IEEE Trans Image Process. (2012) 21:469-80. doi: 10.1109/TIP.2011.2161482

11. Wang Z, Yang Y, Wang Z, Chang S, Yang J, Huang TS. Learning superresolution jointly from external and internal examples. IEEE Trans Image Process. (2015) 24:4359-71. doi: 10.1109/TIP.2015.2462113

12. Yang J, Wright J, Huang TS, Ma Y. Image super-resolution via sparse representation. IEEE Trans Image Process. (2010) 19:2861-73. doi: 10.1109/TIP.2010.2050625

13. Bioucas-Dias JM, Figueiredo MA. A new twist: two-step iterative shrinkage/thresholding algorithms for image restoration. IEEE Trans Image Process. (2007) 16:2992-3004. doi: 10.1109/TIP.2007.909319

14. Rudin LI, Osher S. Total variation based image restoration with free local constraints. In: IEEE International Conference on Image Processing, 1994. ICIP-94. IEEE (1994). p. 31-5.

15. P. Getreuer, Contour stencils: Total variation along curves for adaptive image interpolation. SIAM J Imaging Sci. (2011) 4:954-79. doi: 10.1137/100802785

16. Kim KI, Kwon Y. Single-image super-resolution using sparse regression and natural image prior. IEEE Trans Pattern Anal Mach Intell. (2010) 32:1127-33. doi: 10.1109/TPAMI.2010.25

17. Sun J, Xu Z, Shum H-Y. Image super-resolution using gradient profile prior. In: IEEE Conference on Computer Vision and Pattern Recognition, 2008. CVPR. IEEE (2008). p. 1-8.

18. Fattal R. Image upsampling via imposed edge statistics. ACM Trans Graph. (2007) 26:95. doi: 10.1145/1276377.1276496

19. Lium B, Son S, Kim H, Nah S, Mu K. Enhanced deep residual networks for single image super-resolution. In: The IEEE Conference on Computer Vision and Pattern Recognition (CVPR) Workshops. (2017).

20. Yang W, Zhang X, Tian Y, Wang W, Xue J, Liao Q. Deep learning for single image super-resolution: a brief review. IEEE Trans Multimedia. (2019) 21:3106-21. doi: 10.1109/TMM.2019.2919431

21. Wang Y, Wang L, Wang H, Li P. End-to-end image super-resolution via deep and shallow convolutional networks. IEEE Access. (2019) 7:31959-70. doi: 10.1109/ACCESS.2019.2903582

22. Li Z, Yang J, Liu Z, Yang X, Jeon G, Wu W. Feedback network for image super-resolution. In: IEEE Conference on Computer Vision and Pattern Recognition CVPR. Long Beach, CA: IEEE (2019). doi: 10.1109/CVPR.2019. 00399

23. Ranchin T, Wald L. Fusion of high spatial and spectral resolution images: the ARSIS concept and its implementation. Photogram Eng Remote Sens. (2000) 66:49-61.

24. Loncan L. de Almeida LB, Bioucas-Dias JM, Briottet X, Chanussot J, Dobigeon $\mathrm{N}$, et al. Hyperspectral pansharpening: a review. IEEE Geosci Remote Sens Mag. (2015) 3:27-46. doi: 10.1109/MGRS.2015.2440094

25. Vivone G, Alparone L, Chanussot J, Dalla Mura M, Garzelli A, Licciardi GA, et al. A critical comparison among pansharpening algorithms. IEEE Trans Geosci Remote Sens. (2015) 53:2565-86. doi: 10.1109/TGRS.2014.2361734
26. Shettigara V. A generalized component substitution technique for spatial enhancement of multispectral images using a higher resolution data set. Photogramm Eng Remote Sens. (1992) 58:561-7.

27. Shah VP, Younan NH, King RL. An efficient pan-sharpening method via a combined adaptive PCA approach and contourlets. IEEE Trans Geosci Remote Sens. (2008) 46:1323-35. doi: 10.1109/TGRS.2008.916211

28. Tu T-M, Su S-C, Shyu H-C, Huang PS. A new look at IHSlike image fusion methods. Inform Fusion. (2001) 2:177-86. doi: 10.1016/S1566-2535(01)00036-7

29. Carper JW, Lillesand TM, Kiefer RW. The use of intensityhue- saturation transformations for merging spot panchromatic and multispectral image data. Photogramm Eng Remote Sens. (1990) 56:459-67.

30. Chavez P, Sides SC, Anderson JA. Comparison of three different methods to merge multiresolution and multispectral data: landsat TM and SPOT panchromatic. Photogramm Eng Remote Sens. (1991) 57:295-303.

31. Gillespie AR, Kahle AB, Walker RE. Color enhancement of highly correlated images. I. Decorrelation and HSI contrast stretches. Remote Sens Environ. (1986) 20:209-35. doi: 10.1016/0034-4257(86)90044-1

32. Laben CA, Brower BV. Process for Enhancing the Spatial Resolution of Multispectral Imagery Using Pan-Sharpening. US Patent 6011875 (2000).

33. J. Liu, Smoothing filter-based intensity modulation: a spectral preserve image fusion technique for improving spatial details. Int J Remote Sens. (2000) 21:3461-72. doi: 10.1080/014311600750037499

34. Aiazzi B, Alparone L, Baronti S, Garzelli A, Selva M. An MTF-based spectral distortion minimizing model for pan-sharpening of very high resolution multispectral images of urban areas. In: 2nd GRSS/ISPRS Joint Workshop on Remote Sensing and Data Fusion over Urban Areas. IEEE (2003). p. 90-4.

35. Aiazzi B, Alparone L, Baronti S, Garzelli A, Selva M. MTF-tailored multiscale fusion of high-resolution MS and pan imagery. Photogramm Eng Remote Sens. (2006) 72:591-6. doi: 10.14358/PERS.72.5.591

36. Vivone G, Restaino R, Dalla Mura M, Licciardi G, Chanussot J. Contrast and error-based fusion schemes for multispectral image pansharpening. IEEE Geosci Remote Sens Lett. (2014) 11:930-4. doi: 10.1109/LGRS.2013.2281996

37. Otazu X, González-Audícana M, Fors O, Núñez J. Introduction of sensor spectral response into image fusion methods. Application to waveletbased methods. IEEE Trans Geosci Remote Sens. (2005) 43:2376-85. doi: 10.1109/TGRS.2005.856106

38. Hou L, Zhang X. Pansharpening image fusion using cross-channel correlation: a framelet-based approach. J Math Imaging Vis. (2016) 55:36-49. doi: $10.1007 / \mathrm{s} 10851-015-0612-\mathrm{x}$

39. He X, Condat L, Bioucas-Dias JM, Chanussot J, Xia J. A new pansharpening method based on spatial and spectral sparsity priors. IEEE Trans Image Process. (2014) 23:4160-74. doi: 10.1109/TIP.2014.2333661

40. Deng L-J, Guo W, Huang T-Z. Single-image super-resolution via an iterative reproducing kernel hilbert space method. IEEE Trans Circ Syst Video Technol. (2016) 26:2001-14. doi: 10.1109/TCSVT.2015.2475895

41. Wahba G, Spline Models for Observational Data. Society for Industrial and Applied Mathematics. Philadelphia, PA: Siam (1990). doi: $10.1137 / 1.9781611970128$

42. Irani M, Peleg S. Super resolution from image sequences. In: 10th International Conference on Pattern Recognition. IEEE (1990). p. 115-20.

43. Deng L-J, Vivone G, Guo W, Dalla Mura M, Chanussot J. A variational pansharpening approach based on reproducible kernel hilbert 12 space and heaviside function. IEEE Trans Image Process. (2018). doi: 10.1109/ICIP.2017.8296338

44. Duchon J. Splines minimizing rotation-invariant semi-norms in Sobolev spaces. In: Constructive theory of Functions of Several Variables. Berlin: Springer (1977). p. 85-100. doi: 10.1007/BFb0086566

45. Meinguet J. Multivariate interpolation at arbitrary points made simple. Z Angew Math Phys. (1979) 30:292-304. doi: 10.1007/BF0160 1941

46. Kainen P, Kůrková V, Vogt A. Best approximation by linear combinations of characteristic functions of half-spaces. J Approx Theory. (2003) 122:151-9. doi: 10.1016/S0021-9045(03)00072-8

47. Arthur D, Vassilvitskii S. k-means++: the advantages of careful seeding. In: Proceedings of the Eighteenth Annual ACM-SIAM Symposium on Discrete Algorithms. Society for Industrial and Applied Mathematics. (2007). p. 1027-35. 
48. Kowalski M. Sparse regression using mixed norms. Appl Comput Harm Anal. (2009) 27:303-24. doi: 10.1016/j.acha.2009.05.006

49. Boyd S, Parikh N, Chu E, Peleato B, Eckstein J. Distributed optimization and statistical learning via the alternating direction method of multipliers. Found Trends Mach Learn. (2011) 3:1-122. doi: 10.1561/2200000016

50. Eckstein J, Bertsekas DP. On the Douglas-Rachford splitting method and the proximal point algorithm for maximal monotone operators. Math Program. (1992) 55-3:293-318. doi: 10.1007/BF01581204

51. Rousseeuw P. Silhouettes: a graphical aid to the interpretation and validation of cluster analysis. J Comput Appl Math. (1987) 20:53-65. doi: 10.1016/0377-0427(87)90125-7
Conflict of Interest: The authors declare that the research was conducted in the absence of any commercial or financial relationships that could be construed as a potential conflict of interest.

Copyright (c) 2020 Lartey, Guo, Zhu and Grohnfeldt. This is an open-access article distributed under the terms of the Creative Commons Attribution License (CC BY). The use, distribution or reproduction in other forums is permitted, provided the original author(s) and the copyright owner(s) are credited and that the original publication in this journal is cited, in accordance with accepted academic practice. No use, distribution or reproduction is permitted which does not comply with these terms. 